DRAFT

\title{
Fermions in Quantum Gravity
}

\author{
Hugo A. Morales-Técotl ${ }^{1}$ and Carlo Rovelli ${ }^{2}$ \\ ${ }^{1}$ SISSA, Strada Costiera 11, Trieste, Italy \\ ${ }^{2}$ Department of Physics, University of Pittsburgh, Pittsburgh,15260 U.S.A. \\ ${ }^{2}$ Dipartimento di Fisica, Universita' di Trento. INFN, Sezione di Padova, Italia.
}

\begin{abstract}
We study the quantum fermions + gravity system, that is, the gravitational counterpart of QED. We start from the standard Einstein-Weyl theory, reformulated in terms of Ashtekar variables; and we construct its non-perturbative quantum theory by extending the loop representation of general relativity.

To this aim, we construct the fermion equivalent to the loop variables, and we define the quantum theory as a representation of their Poisson algebra. Not surprisingly, fermions can be incorporated in the loop representation simply by including open curves into "Loop space", as expected from lattice Yang-Mills theory. We explicitely construct the diffeomorphism and hamiltonain operators. The first can be fully solved as in pure gravity. The second is constructed by using a background-independent regularization technique. The theory retains the clean geometrical features of the pure quantum gravity. In particular, the hamiltonian constraint admits the same simple geometrical interpretation as its pure gravity counterpart: it is the operator that shifts curves along themselves ("shift operator"). Quite surprisingly, we believe, this simple action codes the full dynamics of the interacting fermion-gravity theory.

To unravel the dynamics of the theory we study the evolution of the fermiongravity system in the physical-time defined by an additional coupled ("clock"-) scalar field. We explicitely construct the Hamiltonian operator that evolves the system in this physical time. We show that this Hamiltonian is finite, diffeomorphism invariant, and has a simple geometrical action confined to the intersections and the end points of the "loops". The quantum theory of fermions+gravity evolving in the clock time is finally given by the combinatorial and geometrical action of this Hamiltonian on a set of graphs with a finite number of end points. This geometrical action defines the "topological Feynman rules" of the theory.
\end{abstract}




\section{Towards Quantum Gravitational Dynamics}

\subsection{Motivations}

The theoretical description of the Quantum Electromagnetic Field was developed in the thirties, shortly after the birth of quantum theory. In spite of some immediate successes as the justification of the existence of photons, it is not untill the construction of the full quantum theory of the fermions + electromagnetism system, nameley QED, that the full power of this theory became clear. Recently, a certain number of advances toward the construction of Quantum General Relativity (GR) have been achieved [1, 2, 3, 4, 5] (for an introduction see [6, 7, 8]). However, we suspect that this theory too will express its full physical value only when a fully interacting matter + gravity theory is constructed, and, in particular, when the realistic fermions+gravity theory is constructed. By analogy with QED, we shall denote the quantum theory of fermions + gravity as Quantum Gravitational Dynamics, or QGD.

There is a number of reasons for suspecting that matter couplings are needed for clarifying the Quantum Gravity puzzle. The first of these, is that it is very difficult to write fully gauge invariant quantities on the phase space of General Relativity alone, due to diffeomorphism invariance [1], 9. Equivalently, it is extremely difficult to imagine well-defined experiments to be performed on the gravitational field alone. Thus, in the pure gravity case we are in the funny situation of constructing a theory, but not being sure of what precisely should we ask to the theory - a situation quite familiar, we believe, to anybody working in non-perturbative quantum gravity. On the other side, diffeomorphism invariant quantities, as well as realistic experiments described by those quantities, can be constructed in a relative simple fashion in the presence of dynamically coupled matter [9, 10, 11]. For instance, the Solar System, or a binary pulsar emitting gravitational radiation, are examples of matter + gravity systems that we understand well as far as measurements are concerned: we know exactly which meaningfull observables we can measure. If these observables could be measured with Planck-scale sensitivity, then these systems could be seen as quantum gravity laboratories. Clearly, we need a matter + gravity quantum theory in order to describe them theoretically.

A second reason for coupling matter to quantum gravity, is given by the peculiarity of the non-perturbative quantum theory of gravity. In the Loop Representation [1, 12], the theory has a characteristic geometrical structure: Quantum states of gravity are classified by Knot Theory [1], and the dynamics can be represented in a fully combinatorial-algebraic fashion on Knot Space [5]. These features are not accidental, but rather are consequences of diffeomorphism invariance; equivalently, they are related to the fact that the Loop Representation is a genuine background independent quantum field theory. Since the theory relies on these geometrical structure, it is mandatory to check whether 
these structures are lost when further fields are coupled. If so, doubts could be cast on the value of the Loop Representation of Quantum GR.

\subsection{Introducing fermions in the loop representation}

Motivated by the considerations above, we have studied the fermions + gravity system, or QGD, in the Loop Representation. The choice of fermions is motivated by realism, but also by the fact that they are very natural objects in the Ashtekar formalism. The study of the gravitational interaction of fermions in the light of General Relativity, goes back to Dirac and Sciama [13, and has more recently been investigated by Nelson and Teitelboim [14] using a canonical approach. The theory has been formulated in terms of Ashtekar variables in ref. [16]; we review this formalism below. We then construct the quantum theory following the lines along which the quantum theory of pure General Relativity was constructed. We define the natural extension of the loops observables to fermions (these are given by a parallel transport operator associated to an open curve contacted with fermions sited at the end points of the curve), study their Poisson algebra, and define the quantum theory as a linear representation of this algebra. In analogy with the pure gravity case, we show that the resulting representation can also be heuristically obtained from a naive Shrödinger-like representation by means of a (ill defined) Loop Transform.

The loop representation of QGD turns out to be a very natural extension of the pure gravity case, obtained by including open curves into Loop Space. This is certainly not surprising, since the kinematics of the loop representation can be seen as the continuum version of the Wilson-Kogut construction in lattice Yang-Mills theory [17], and from the work of Gambini and collaborators [12] where fermions are represented by the end points of open lines of flux on the lattice. In the rest of the paper, we will denote both open and closed curves as loops, disregarding consistency with the dictionary. It is not difficult to solve the diffeomorphism constraint on the resulting state space. The complete classification of the solutions is given by a generalization of the Knot Classes of the pure gravity case - the new classes include graphs with an arbitrary number of intersections and open ends. Thus, quantum states of QGD admit the same kind of topological description as the states of pure Quantum GR, contrary to the fear that this aspect of the Loop Representation could be lost in presence of matter couplings. We view this as an encouraging result, though a result that could have been anticipated.

On the other side, the results we obtain about the dynamics of the theory are unexpected and, we believe, rather surprising. The dynamics is given by the hamiltonian constraint, which includes the fermion kinetic energy and the fermion-gravity interaction. We construct in this paper the corresponding quantum operator, and its action on the loops turns out to have an extremely simple geometrical interpretation: The hamilonian constraint operator essentially "shifts" loops along their tangent. This same simple geometrical action 
of the hamiltonian constraint was recognized in the context of pure gravity in ref.[1]. The surprising result here is that the very same action, extended to open loops, codes the kinetic fermion energy and the fermions-gravity interaction. This was first noticed by one of us in ref. 18].

\subsection{Introducing a clock}

While suggestive, the above construction is not fully satisfactory for three reasons. First there is a divergence in the action of the hamiltonian operator which is difficult to control. Second, in spite of the simplicity of the action of the hamiltonian operator, we have not been able to solve the corresponding quantum constraint equation. Third, the presence of the fermions does not takes us completely out from the difficulties of constructing gauge invariant observables: it simplifies the task of finding three-dimensional diffeomorphism invariant quantities, but it does not help with the problem of finding quantities that commute with the hamiltonain constraint. Thus, the actual physical content of the theory is still quite unaccessible, as it is in pure gravity.

To face these problems, we take one further step. We combine our results on the fermions with the results obtained in ref. [5]. In that paper, the idea was proposed to unravel the dynamics of quantum gravity by coupling a scalar field, which could behave as a clock-field, following a long tradition [11] of ideas of using matter for simplifying the gravitational theory analysis (see $\sqrt{9}, 10$. 19, 20, 21, 22]). It is shown in Ref. [5] that by a suitable gauge fixing one can express the dynamics of gravity as intrinsic evolution with respect to the intrinsic time (or physical time) defined by the scalar field. This evolution is explicitely generated on the state space by a hamitonian operator $\hat{H}$. Here, we extend this construction to fermions. Namely, we consider the generally covariant gravity+fermions+scalar field system, we solve with respect to the scalar field, so that the hamiltonian constraint disappears from the theory, and is replaced by a genuine diffeomorphism invariant hamiltonian that evolves both gravity and fermions in the scalar field clock time. We explicitely construct the quantum hamiltonian operator $\hat{H}$ (as opposed to hamiltonian constraint operator), by making use of the regularization techniques on manifolds that have recently been introduced [2] in quantum gravity. In the fermion sector, we recover in this way the simple action described above, namely the shift of the loops along themselves. However, now the resulting operator is fully diffeomorphism invariant, and finite .

\section{$1.4 Q G D$}

The resulting QGD is then given by a set of quantum states represented by graphs with a finite number of intersections and open ends, and by an Hamiltonian operator that acts in a simple geometrical and combinatorial fashion on these graphs. Matrix elements of this Hamiltonian can be interpreted as first 
order transition amplitudes between the graph states in a time dependent perturbation expansion in the clock time. The explicit computation is complicated by the need of extracting the square root of an infinite matrix, a task we expect could be solved order by order. In the present paper, we only begin the explicit computation of matrix elements of the operator.

The picture of Quantum Gravitational Dynamics that begins to emerge from this construction has a simple and perhaps appealing general structure: A graph with two open ends, say, represents two fermions interacting gravitationally among themselves, and with the surrounding gravitational field. With the machinery developed in this paper we could (at least in principle) follow the quantum evolution of this system in clock time.

The paper is organized as follows. In section 2, we review the classical Einstein-Weyl theory in Ashtekar form, and we introduce the loop variables and their extension to fermions. In section 3, we define the quantum theory, and we discuss and solve the quantum diffeomorphism constraint. In section 4, we give a preliminary discussion of the Hamiltonian quantum constraint and its surprising simplicity - the fully consistent construction needs the clock-field. In section 5, we recall the main ideas of the clock-field construction, we define the gravity+fermions+clock- field theory, we study the regularization of the Hamiltonian, and we obtain the rigorous form of the Hamiltonian operator. In section 6, we describe resulting structure of QGD, we collect a certain number of comments on the general construction, we discuss the lines along which the theory should be further developed, and we summarize our results.

The signature of the spacetime metric is $(-+++)$. Throughout units are used in which $G=c=\hbar=1$, except in the last section.

\section{Einstein-Weyl theory in Ashtekar form}

We consider the system formed by general relativity and a Weyl fermion field. We describe general relativity in terms of Ashtekar variables [23; and the fermion in terms of a two- component, massless spinor field $\psi^{A}(x), A=1,2$.

A word should be spent concerning the coupling between the fermions and gravity. Matter spinor fields coupled to gravity in the Ashtekar's formalism have been studied in refs. [16] (see also [24]). Ashtekar et.al. have added a quartic terms to the minimally coupled fermion-gravity action in order to get the equations of motion of the Einstein-Weil torsion-free theory. As made clear in [25], the minimally coupled action without quartic terms, yelds the EinsteinCartan theory, in which fermions act as a source of (non-propagating) torsion. 巴. For simplicty, we consider here the minimally coupled theory. The system

\footnotetext{
${ }^{1}$ In the theory with the quartic interaction one can naturally define a connection which is the sum of the torsion-free selfdual connection, and a matter generated term; the equations of motion are then structurally equal to the Einstein-Weil equations, but with the selfdual connection replaced by the full connection. See 25 for more details.
} 
represents for instance neutrinos in a dynamical spacetime. We expect that the extension to Dirac fermions, and to the theory with the quartic interaction should be straight forward.

In recent years it has become conventional to work with (classical) spinor fields which are Grassmann valued. We (reluctantly) decided to follow this practice because it simplifies the bookkeeping of the signs in promoting Poisson brackets to (anti-) commutators (since we do not use canonically conjugate variables as basics observables for the quantization). Thus, we assume the fields $\psi^{A}(x)$ anticommute.

Spacetime is taken as a manifold $M$ with the topology $\Sigma \times \mathbb{R}$, with $\Sigma$ compact. We indicate spacetime indices (four dimensional as well as three dimensional) with lower case latin letters. Over and under tildes denote densities of weight +1 or -1 respectively (on $M$ as well as on $\Sigma$ ). Since we are dealing with spinors, it is natural to adopt the spinor version of the Ashtekar formalism. The gravitational variables are then the spacetime $S L(2, \mathbb{C})$ soldering form ${ }^{4} \sigma^{a}{ }_{A}{ }^{\prime}(x)$, that is the spinor form of the tetrad field, and the $S L(2, \mathbb{C})$ Ashtekar connection [23, which has only unprimed indices, ${ }^{4} A_{a A}^{B}(x)$, and which is interpreted as the (internal-) selfdual part of the spin connection. We give the explicit relation with the vector quantities below. The Lagrangian of the system is [16, 26, 27]

$$
\mathcal{L}_{\text {Einstein-Weyl }}=\left({ }^{4} \sigma\right)^{4} \sigma^{a}{ }_{A}{ }^{\prime} 4 \sigma^{b}{ }_{B A^{\prime}}{ }^{4} F_{a b}{ }^{A B}+\sqrt{2}\left({ }^{4} \sigma\right)^{4} \sigma_{A A^{\prime}}^{a} \bar{\psi}^{A^{\prime} 4} \mathcal{D}_{a} \psi^{A} .
$$

In order to construct the quantum theory, we set the theory in canonical form. This can be done in a fully covariant way by taking the space of the solutions of the equations of motion as the phase space, and defining the appropriate symplectic struture over it. However, we follow here the equivalent, but simpler and more conventional, approach, based on the $3+1$ decomposition, even if it obscures the natural general covariance of the hamiltonian formulation (see for instance 28, 29]).

The torsion-free derivative operator compatible with ${ }^{4} \sigma^{a}{ }_{A}{ }^{\prime}$ will be denoted $\nabla$, while the derivative operator associated to the selfdual connection ${ }^{4} A_{a A}{ }^{B}$ is denoted by ${ }^{4} \mathcal{D}$. Let $t$ be a function with nowhere vanishing gradient, whose level surfaces $\Sigma_{t}$ are diffeomorphic to $\Sigma$. We denote the (three dimensional) pull back to $\Sigma_{t}$ of four dimensional quantities with the same notation as the four dimensional quantity, taking away the superscript " 4 ", if present. Let also $t^{a}$ be a vector field with affine parameter $t$, i.e. $t^{a} \nabla_{a} t=1$. Given a soldering form ${ }^{4} \sigma_{A A^{\prime}}^{a}$ such that each $\Sigma_{t}$ is spacelike, take the normal to $\Sigma_{t}$ as $n^{a}: n^{a} n_{a}=-1$. The induced metric on $\Sigma_{t}$ is denoted $q_{a b}:=g_{a b}+n_{a} n_{b}$. Thereby every tensor field can be decomposed into its parts on and orthogonal to $\Sigma_{t}$. In particular, $t^{a}$ gives us the Lapse and Shift: $t^{a}=N n^{a}+N^{a}$. Unprimed $S L(2, \mathbb{C})$ spinors, on $M$, and $S U(2)$ spinors, on $\Sigma$, can be related as follows. One defines the hermitian metric in the spinor space, $G^{A A^{\prime}}:=i \sqrt{2} n^{A A^{\prime}}\left(=\bar{G}^{A^{\prime} A}\right)$, where $n^{A A^{\prime}}:=\sigma_{a}{ }^{A A^{\prime}} n^{a}$. The primed $S L(2, \mathbb{C})$ spinors define a hermitian conjugation 
operation on the unprimed ones through $\left(\psi^{\dagger}\right)_{A}:=G_{A}^{A^{\prime}} \bar{\psi}_{A^{\prime}}$. The hermitian conjugate of $\psi_{A}$. The soldering form for $S U(2)$ spinors

$$
\sigma_{A B}^{a}:=-i \sqrt{2}{ }^{4} \sigma_{(A}^{a}{ }^{A^{\prime}} n_{B) A^{\prime}},
$$

is hermitian $\left(\sigma_{A B}^{a}\right)^{\dagger}=\sigma_{A B}^{a}$, and traceless $\sigma_{A}^{a}{ }_{A}=0$; it provides, locally, an isomorphism between $T \Sigma$ and second rank, trace-free, hermitian spinors $\gamma_{A B}$. In particular $q^{a b}=-\operatorname{Tr}\left(\sigma^{a} \sigma^{b}\right)$.

The configuration variables can be taken as $A_{a A}{ }^{B}$ and $\psi^{A}$, whereas the correspondent canonically conjugated momenta are $-i \sqrt{2} \widetilde{\sigma}_{A}^{a}{ }_{A}:=-i \sqrt{2}(\sigma) \sigma_{A}^{a}{ }_{A}$ and $\widetilde{\pi}_{A}:=-i(\sigma)\left(\psi^{\dagger}\right)_{A}$. The action of the Einstein-Weyl system becomes:

$$
\begin{aligned}
S_{\text {Einstein-Weyl }}= & \int d t \int_{\Sigma_{t}} d^{3} x \widetilde{\pi}_{A} \mathcal{L}_{t} \psi^{A}+\operatorname{Tr}\left[\left(-i \sqrt{2} \widetilde{\sigma}^{b}\right) \mathcal{L}_{t} A_{b}\right]+ \\
& +\left(t \cdot{ }^{4} A^{A B}\right) \widetilde{G}_{A B}+N^{a} \widetilde{V}_{a}+\underset{\sim}{N} \widetilde{\widetilde{S}}
\end{aligned}
$$

Where $\mathcal{L}_{t}$ is the Lie derivative along $t^{a}$. Here

$$
\begin{aligned}
\widetilde{G}_{A B} & =-i \sqrt{2} \mathcal{D}_{b} \widetilde{\sigma}_{A B}^{b}+\widetilde{\pi}_{(A} \psi_{B)} \\
\widetilde{V}_{a} & =-i \sqrt{2} \operatorname{Tr}\left(\widetilde{\sigma}^{b} F_{a b}\right)-\widetilde{\pi}_{A} \mathcal{D}_{a} \psi^{A} \\
\widetilde{\widetilde{S}} & =-\operatorname{Tr}\left(\widetilde{\sigma}^{a} \widetilde{\sigma}^{b} F_{a b}\right)+i \sqrt{2} \widetilde{\sigma}_{A}^{a} \widetilde{\pi}_{B} \mathcal{D}_{a} \psi^{A} .
\end{aligned}
$$

These are the Gauss, vector and scalar constraints, respectively, for the EinsteinWeyl system as given in terms of Ashtekar variables. Finally, the theory is defined by the conventional reality conditions which pick the real sector of the phase space to which classical general relativity belongs. We will not discuss reality conditions in this paper.

The symplectic structure one arrives at is

$$
\begin{aligned}
\left\{\widetilde{\sigma}^{a}{ }_{A B}(x), A_{b}{ }^{C D}(y)\right\} & =-\frac{i}{\sqrt{2}} \delta^{3}(x, y) \delta_{b}{ }^{a} \delta_{(A}{ }^{C} \delta_{B)}{ }^{D}, \\
\left\{\widetilde{\pi}_{A}(x), \psi^{B}(y)\right\} & =-\delta^{3}(x, y) \delta_{A}{ }^{B} .
\end{aligned}
$$

The relation between these canonical variables and the vectorial Ashtekar variables (see 6), namely the densitized triad field $\tilde{E}^{a i}(x)$ and the $S O(3)$ connection $A_{a}^{i}(x)$, is given by

$$
\widetilde{\sigma}_{A}^{a}{ }^{B}(x)=\frac{-i}{\sqrt{2}} \tilde{E}^{a i}(x) \sigma_{i A}{ }^{B}, \quad A_{a A}{ }^{B}(x)=\frac{-i}{2} A_{a}^{i}(x) \sigma_{i A}{ }^{B}
$$

where $i=1,2,3$ and $\sigma_{i}$ are the Pauli matrices.

With this simplectic structure, the constraints can be interpreted as generators of gauge transformations, spatial diffeomorphisms and the very dynamics. 
This is shown, together with the fact that they form a Poisson algebra, in [Ashtekar et.al. ] by smearing the constraints as

$$
\begin{aligned}
G_{T} & \equiv-\int_{\Sigma} d^{3} x T^{B A} \widetilde{G}_{A B} \\
D_{v} & \equiv \int_{\Sigma} d^{3} x\left[v^{a} \widetilde{V}_{a}-v^{a} A_{a}^{B A} \widetilde{G}_{A B}\right] \\
H_{N} & \equiv i \sqrt{2} \int_{\Sigma} d^{3} x \underset{\sim}{N} \widetilde{\widetilde{S}}
\end{aligned}
$$

$T^{A B}, v^{a}, N$ are arbitrary test fields.

\subsection{Classical fermion paths}

Non-perturbative quantum General Relativity is constructed in terms of the loop variables [1]

$$
\begin{aligned}
T[\alpha] & :=1 / \text { over } 2 U_{A}^{A}[\alpha] \\
T^{a}[\beta](s) & :=\sqrt{2} U_{A}^{B}[\beta](s) \widetilde{\sigma}_{B}^{a}{ }^{A}(\beta(s)) .
\end{aligned}
$$

We indicate loops by greek letters. A loop is here a closed continuous piecewise analytical curve in $\Sigma, \alpha: S_{1} \rightarrow \Sigma$; and $s \in[0,1]$ is the parameter along the loop: $\alpha: s \mid \rightarrow \alpha^{a}(s)$ (We identify the values $s$ and $s+1$ ). We indicate by $U_{A}^{B}[\alpha](s)$ the parallel transport $S L(2, \mathbb{C})$ matrix of the Ashtekar connection around the loop $\alpha$, starting from the parameter value $s$; that is, the path order exponential of the line integral of the connection around the loop.

$$
U_{A}^{B}[\beta](s):=P \exp \left\{\int_{s}^{s+1} d s \frac{d \alpha^{a}(s)}{d s} A_{a A}{ }^{B}\right\} .
$$

In order to construct the extension of the loop representation to fermions, we want to generalize these loop variables to the presence of the spinor field. We want to define objects invariant under the action of the Gauss constraint. Looplike variables in theories with connections involving fermions, have been dealt with by Gambini and collaborators [12] for Yang-Mills, and by Kim et.al. [30], for $2+1$ gravity. For earlier related ideas, see 17, 31. We follow here the lines of [32]. Let us consider piecewise differentiable continuous open curves in $\Sigma$. As we said in the introduction, we will call these open lines as loops, certainly with an abuse of terminology; and we denote them too by means of greek letters: $\alpha:(0,1) \rightarrow \Sigma$; and $\alpha: s \mid \rightarrow \alpha^{a}(s)$. We denote as $\alpha_{i}$ and $\alpha_{f}$ the initial and final point of the loop, namely:

$$
\begin{aligned}
\alpha_{i} & :=\alpha(0), \\
\alpha_{f} & :=\alpha(1) .
\end{aligned}
$$


If $\alpha_{f}=\beta_{i}$, we denote the open line obtained joining $\alpha$ and $\beta$ as $\alpha \cdot \beta$; that is

$$
\begin{gathered}
{[\alpha \cdot \beta](s):=\alpha(2 s), \quad \text { if } s \in[0,1 / 2]} \\
{[\alpha \cdot \beta](s):=\beta(2 s-1), \quad \text { if } s \in[1 / 2,1] .}
\end{gathered}
$$

We then define

$$
\begin{aligned}
X[\alpha] & :=\psi^{A}\left(\alpha_{i}\right) U_{A}^{B}[\alpha] \psi_{B}\left(\alpha_{f}\right) \\
Y[\alpha] & :=\widetilde{\pi}^{A}\left(\alpha_{i}\right) U_{A}^{B}[\alpha] \psi_{B}\left(\alpha_{f}\right) .
\end{aligned}
$$

$X$ and $Y$, are parametrized by open curves. They are defined as path integral exponentials of the Ashtekar connection along these curves, with spinors variables attached to the end points. They are clearly $S U(2)$-invariant. Other important properties of the $X$ and $Y$ variables are the following.

1. They are invariant under a positive derivative monotonic reparametrization of the open loops.

2. $X$ is invariant under inversion of the open loop, $X\left[\alpha^{-1}\right]=X[\alpha]$. This important property follows from the fact the fermions are Grassman variables. In fact:

$$
\begin{aligned}
X\left[\alpha^{-1}\right] & =\psi^{A}\left(\alpha_{i}^{-1}\right) U_{A}^{B}\left[\alpha^{-1}\right] \psi_{B}\left(\alpha_{f}^{-1}\right) \\
& =\psi^{A}\left(\alpha_{f}\right) U_{A}{ }^{B}\left[\alpha^{-1}\right] \psi_{B}\left(\alpha_{i}\right) \\
& =-\psi^{A}\left(\alpha_{f}\right) U_{A B}\left[\alpha^{-1}\right] \psi^{B}\left(\alpha_{i}\right) \\
& =+\psi^{A}\left(\alpha_{f}\right) U_{B A}[\alpha] \psi^{B}\left(\alpha_{i}\right) \\
& =-\psi^{B}\left(\alpha_{i}\right) U_{B A}[\alpha] \psi^{A}\left(\alpha_{f}\right) \\
& =+\psi^{B}\left(\alpha_{i}\right) U_{B}{ }^{A}[\alpha] \psi_{A}\left(\alpha_{f}\right) \\
& =+\psi^{A}\left(\alpha_{i}\right) U_{A}^{B}[\alpha] \psi_{B}\left(\alpha_{f}\right) \\
& =X[\alpha]
\end{aligned}
$$

In the third and sixth line we have used the spinor index property $\xi^{A} \rho_{A}=$ $-\xi_{A} \rho^{A}$. In the fourth line we have used the parallel propagator property $U_{A B}[\alpha]=-U_{B A}\left[\alpha^{-1}\right]$ (recall that if the parallel propagator $U_{A}{ }^{B}[\alpha]$ is the identity, then $U_{A B}[\alpha]=\epsilon_{A B}$ ). In the fifth line, we have switched the two fermions, gaining a minus sign due to their Grassmanian character.

3. Retracing identity. As their closed loops counterparts, the fermionic loop variables $X$ and $Y$ satisfies the retracing and spinor identities that follows from their being defined in terms of parallel propagators of an $S U(2)$ connection. For instance, if $\beta_{f}=\gamma_{i}=\delta_{i}$, then $X\left[\beta \cdot \gamma \cdot \gamma^{-1} \cdot \delta\right]=X[\beta \cdot \delta]$.

4. Spinor identity. The following notation is useful. Let $\alpha$ be an (oriented) open line. We define

$$
\alpha^{A}:=\psi^{B}\left(\alpha_{i}\right) U_{B}^{A}[\alpha]
$$


Then, if $\alpha_{f}=\beta_{f}$, we can write

$$
X\left[\alpha \cdot \beta^{-1}\right]=\alpha^{A} \beta_{A}
$$

Now, consider a point $p$ where 4 lines terminate, that is $\alpha_{f}=\beta_{f}=\gamma_{f}=$ $\delta_{f}=p$. There are three possible ways of connecting these four lines to form two gauge invariant $X$ variables:

$$
\begin{aligned}
& X\left[\alpha \cdot \gamma^{-1}\right] X\left[\delta \cdot \beta^{-1}\right]=\alpha_{A} \gamma^{A} \delta_{B} \beta^{B}=\alpha^{A} \beta^{B} \gamma^{C} \delta^{D} \epsilon_{A C} \epsilon_{D B} \\
& X\left[\alpha \cdot \delta^{-1}\right] X\left[\gamma \cdot \beta^{-1}\right]=\alpha_{A} \delta^{A} \gamma_{B} \beta^{B}=\alpha^{A} \beta^{B} \gamma^{C} \delta^{D} \epsilon_{A D} \epsilon_{B C} \\
& X\left[\alpha \cdot \beta^{-1}\right] X\left[\gamma \cdot \delta^{-1}\right]=\alpha_{A} \beta^{A} \gamma_{B} \delta^{B}=\alpha^{A} \beta^{B} \gamma^{C} \delta^{D} \epsilon_{A B} \epsilon_{C D} .
\end{aligned}
$$

By using the fundamental spinor identity, which is at the root of the Mandelstam relations

$$
\epsilon_{A B} \epsilon_{C D}+\epsilon_{A D} \epsilon_{B C}+\epsilon_{A C} \epsilon_{D B}=0
$$

we have the fermion version of the spinor identity, namely

$$
X\left[\alpha \cdot \gamma^{-1}\right] X\left[\delta \cdot \beta^{-1}\right]+X\left[\alpha \cdot \delta^{-1}\right] X\left[\gamma \cdot \beta^{-1}\right]+X\left[\alpha \cdot \beta^{-1}\right] X\left[\gamma \cdot \delta^{-1}\right]=0 .
$$

Similarly, it is simple to derive the identitity that refers to the intersection between the open loop $\alpha \cdot \beta$ and the closed loop $\gamma$, where $\alpha_{f}=\beta_{i}=\gamma_{i}=\gamma_{f}$ :

$$
X[\alpha \cdot \beta] T[\gamma]=X[\alpha \cdot \gamma \cdot \beta]+X\left[\alpha \cdot \gamma^{-1} \cdot \beta\right]
$$

5. Fermionic (Grassmann) identities. Since Grassman variables anticommute, and since the fermion field has only two components, if we multiply three or more fields in the same point we obtain zero. It follows that we can have products of $X$ variables with at most two coinciding hands. Thus, for instance, if $\alpha_{i}=\beta_{i}=\gamma_{i}$, then $X[\alpha] X[\beta] X[\gamma]=0$.

6. Gauge observables algebra. Finally, the most important property of the $X, Y, T, T^{a}$ variables is that their Poisson algebra closes. A direct computation yelds

$$
\begin{aligned}
& \{X[\beta], X[\alpha]\}=0 \\
& \{Y[\beta], X[\alpha]\}=\delta^{3}\left(\alpha_{f}, \beta_{i}\right) X[\alpha \cdot \beta]+\delta^{3}\left(\beta_{i}, \alpha_{i}\right) X\left[\alpha^{-1} \cdot \beta\right] \\
& \{Y[\alpha], Y[\beta]\}=\delta^{3}\left(\alpha_{f}, \beta_{i}\right) Y[\alpha \cdot \beta]-\delta^{3}\left(\alpha_{i}, \beta_{f}\right) Y[\beta \cdot \alpha]
\end{aligned}
$$

Whereas the nonvanishing brackets with the $T$ variables are

$$
\begin{aligned}
\left\{T^{a}[\gamma](s), X[\alpha]\right\} & =i \Delta^{a}[\gamma(s), \alpha] \sum_{\mu= \pm 1} \mu X\left[\alpha \#_{s} \gamma^{\mu}\right] \\
\left\{T^{a}[\gamma](s), Y[\alpha]\right\} & =i \Delta^{a}[\gamma(s), \alpha] \sum_{\mu= \pm 1} \mu \widetilde{Y}\left[\alpha \#_{s} \gamma^{\mu}\right]
\end{aligned}
$$


As in the pure gravity, one may define also higher order observables, which will have simple quantum operators associated. For instance we can insert "hands" into the $X$ variable, and so on. In order to treat the dynamics, the following variable will be particularly useful.

$$
Y^{a}[\alpha](s):=\pi^{A}\left(\alpha_{i}\right) U_{A}{ }^{B}[\alpha](0, s) \tilde{\sigma}^{a}{ }_{B}^{C}(\alpha(s)) U_{C}{ }^{D}[\alpha](s, 1) \psi_{D}\left(\alpha_{f}\right),
$$

where the notation $U_{A}^{B}[\alpha](s, t)$ indicates the matrix of the parallel trasport along $\alpha$ from $s$ to $t$. This variable is quadratic in the momenta and will play a role analogous to the role of the $T^{2}$ variable in pure gravity, which is also quadratic in the momenta. Indeed, we will use it for defining the hamiltonian constraint.

Finally, we may introduce a further small simplification in the notation: if the context is clear, we may write $X[\alpha]$ as $T[\alpha]$. Namely we can use the same notation, $T[\alpha]$, to indicate the the trace of the holonomy of $\alpha$ if $\alpha$ is a closed loop, and to indicate the parallel propagator along $\alpha$ sandwhiched between two fermion fields if $\alpha$ is open.

\section{QGD: kinematics}

Let us now begin the construction of the quantum theory. Following the philosophy described in refs. [6, 1, 34, we look for a quantum representation of the classical loop algebra. Rather than simply writing the rappresentation, it is perhaps more instructive to use the Loop Transform, introduced in ref. [1], as an heuristic device to help us in this task. Thus we first consider a "Schrödingerlike", rapresentation of the quantum Einstein-Weyl theory [35]. We consider functionals $\Psi[A, \psi]$ on the configuration space, and we define the canonical coordinates operators $A$ and $\psi$ as multiplicative operators, and the corresponding momentum operators as functional derivative operators

$$
\begin{aligned}
\hat{\tilde{\sigma}}^{a}{ }_{A}^{B}(x) & =\frac{1}{\sqrt{2}} \frac{\delta}{\delta A_{a_{B}^{A}}^{A}(x)}, \\
\hat{\tilde{\pi}}_{A}(x) & =\imath \frac{\delta}{\delta \psi^{A}(x)} .
\end{aligned}
$$

Since we are using this construction only as an heuristic tool to find a possible form for the fermion loops operators, we are not particularly concerned with

mathematical rigor here. We focus on loop states in this representation. These are defined as follows. Given a closed loop $\alpha$, we write

$$
\Psi_{\alpha}[A, \psi]=U[\alpha]_{A}^{A} .
$$

Given an open loop $\alpha$, we write

$$
\Psi_{\alpha}[A, \psi]=\psi^{A}\left(\alpha_{i}\right) U[\alpha]_{A}{ }^{B} \psi_{B}\left(\alpha_{f}\right) .
$$


Given an arbitrary collection $\beta$ of a finite number of open or closed loops $\alpha_{1}, \alpha_{2}, \ldots, \alpha_{n}$, we write

$$
\Psi_{\beta}=\Psi_{\alpha_{1}} \Psi_{\alpha_{2}} \ldots \Psi_{\alpha_{n}} .
$$

Note that we follow here the usual convention of denoting loops (open or closed) and multiple loops (namely collections of a finite number of loops) by means of the same notation, that is greek letters from the begining of the alphabet. The idea of the loop representation is to take the $\Psi_{\beta}$ states as an overcomplete basis of quantum states. We thus introduce the Loop Transform [1] as follows.

$$
\Psi[\beta]:=\int \mathcal{D} A \mathcal{D} \psi \Psi_{\beta}[A, \psi] \Psi[A, \psi] .
$$

For a rigorous mathematical definition of these kind of integrals see the recent work of Ashtekar and Isham, and Ashtekar and Lewandowsky [36] Here $\Psi[A, \psi]$ represents a generic wave functional in the connection representation, and $\Psi[\beta]$ represents its Loop Transform. The novel features entering here come from the inclusion of fermions; states containing no fermions are described exactly as in the pure gravity case [1]. The transform (35) has a well defined meaning on the lattice [?], where the $\Psi_{\beta}$ states are the Wilson-Susskind states. In the lattice case it is possible to show that the transform defines a unitary transformation to a new basis in the Hilbert space of the theory.

Eq. (35) suggests that we may look for a representation of the full WeylEinstein loop algebra on a space of functionals of multiple loops, where the multiple loops are sets of closed as well as open loops. Then the transform gives us immediately the action of the $X$ and $Y$ operators in the loop representation

$$
\begin{aligned}
(\widehat{X}[\alpha] \Psi)[\beta] & =\int \mathcal{D} A \mathcal{D} \psi \Psi_{\beta}[A, \psi](\widehat{X}[\alpha] \Psi)[A, \psi] \\
& =\int \mathcal{D} A \mathcal{D} \psi \Psi_{\beta}[A, \psi] \Psi_{\alpha}[A, \psi] \Psi[A, \psi] \\
& =\int \mathcal{D} A \mathcal{D} \psi \Psi_{\beta \cup \alpha}[A, \psi] \Psi[A, \psi] \\
& =\Psi[\beta \cup \alpha] ;
\end{aligned}
$$

where the union operaton $\cup$ of set theory is well defined between the multiple loop $\beta$ and the single open loop $\alpha$. Thus we have

$$
\widehat{X}[\alpha] \Psi[\beta]=\Psi[\beta \cup \alpha] .
$$

Note that this is the essentially the same action as the action of $T[\alpha]$ in pure gravity. Using the notation suggested as the end of the previous section, we can write

$$
\widehat{T}[\alpha] \Psi[\beta]=\Psi[\beta \cup \alpha]
$$

for open as well as for closed $\alpha$ 's. 
As far as $Y$ is concerned, we have

$$
\begin{aligned}
(\widehat{Y}[\alpha] \Psi)[\beta] & =\int \mathcal{D} A \mathcal{D} \psi \Psi_{\beta}[A, \psi](\widehat{Y}[\alpha] \Psi)[A, \psi] \\
& =\int \mathcal{D} A \mathcal{D} \psi\left(\widehat{Y}[\alpha] \Psi_{\beta}\right)[A, \psi] \Psi[A, \psi] \\
& =\int \mathcal{D} A \mathcal{D} \psi\left(\psi_{B}\left(\alpha_{f}\right) U_{A}^{B}[\alpha] \frac{\delta}{\delta \psi^{A}\left(\alpha_{i}\right)} \Psi_{\beta}\right)[A, \psi] \Psi[A, \psi] \\
& =\sum_{\beta_{f}} \delta^{3}\left(\alpha_{i}, \beta_{f}\right) \Psi[\alpha \cdot \beta]+\sum_{\beta_{i}} \delta^{3}\left(\alpha_{i}, \beta_{i}\right) \Psi\left[\beta \cdot \alpha^{-1}\right] .
\end{aligned}
$$

Here $\sum_{\beta_{i}}$ indicates the sum over all the initial points of the open loops in the multiple loop $\beta$, and $\sum_{\beta_{f}}$ indicates the sum over all the final points of the open loops in the multiple loop $\beta$. We introduce the following notation. We write $\beta_{e}$ to indicate any end point of the multiple loop $\beta$. If $\beta_{e}=\alpha_{i}$, the notation $\beta \cdot{ }_{e} \alpha$ indicates the multiple loops obtained by attaching $\alpha_{i}$ with $\beta_{e}$. Thus we have

$$
\widehat{Y}[\alpha] \Psi[\beta]=\sum_{\beta_{e}} \delta^{3}\left(\alpha_{i}, \beta_{e}\right) \Psi\left[\beta \cdot_{e} \alpha\right] .
$$

In words, the action of the operator $Y[\alpha]$ is simply to attach the open loop $\alpha$ to any open end that happens to be in the point $\alpha_{i}$.

Next, we supplement (37), (40) with the usual quantum $T$-variables in the loop representation [1, 6]. The computation of the quantum commutation relations of the entire set is then straigthforward. The result is that the set of operators $\hat{X}, \hat{Y}, \hat{T}, \hat{T}^{a}$ provides a representation of the classical algebra (27), (28) and the classical $\mathcal{T}$-algebra.

We can also naturally introduce quantum operators corresponding to higher order loop variables [1]. As their pure gravity counterparts, these have a quantum algebra that reduces to the corresponding classical Poisson algebra in the limit in which the Planck constant goes to zero. We write here the quantum operator corresponding to the $Y^{a}$ variable defined above, since it will be used in the construction of the Hamiltonian

$$
\begin{aligned}
Y^{a}[\alpha](s) \Psi[\beta]= & \sum_{\beta_{e}} \delta^{3}\left(\beta_{e}, \alpha_{i}\right) \int_{\beta} d t \dot{\beta}^{a}(t) \delta^{3}(\alpha(s), \beta(t)) \\
& {\left[\Psi\left(\alpha * *_{e, t}^{+} \beta\right)+\Psi\left(\alpha * *_{e, t}^{-} \beta\right)\right]=} \\
= & \sum_{\beta_{e}} \delta^{3}\left(\beta_{e}, \alpha_{i}\right) \int_{\beta} d t \dot{\beta}^{a}(t) \delta^{3}(\alpha(s), \beta(t)) \\
& \sum_{q= \pm} \Psi\left(\alpha * *_{e, t}^{q} \beta\right)
\end{aligned}
$$

Here we have indicated by $\alpha * *_{e, t}^{+} \beta$ and $\left.\alpha * *_{e, t}^{-} \beta\right)$ the two loops obtained by joining the $\beta_{e}$ end point of $\beta$ with the point $\alpha_{i}$, and rerooting the intersection 
$\alpha(t)=\beta(s)$ in the two possible ways. Note the plus sign between the two terms, which will play an important role in what follows. The expression (41) can be obtained for instance from the loop transform. To determine the correct overall coefficient and sign, an accurate computation with the $\mathrm{SU}(2)$ index algebra is needed. Note however, that the relative plus sign between the two terms in parenthesis is forced by symmetry, since neither of the two can be preferred.

\subsection{Diffeomorphisms and diff-invariant states}

The classical vector constraint generates spatial diffeomorphisms when acting on gauge invariant objects. This is also true in the quantum theory providing the correct ordering of the vector constraint quantum operator is chosen. Precisely as in the pure gravity theory, there are several equivalent ways for reaching this conclusion:

1. As suggested by Isham 34, the vector constraint can simply be defined as the generator of the natural action of the diffeomorphism group on the space of the open and closed loops. The commutator algebra of these generators among themselves and with all the other operators in the theory, then, reproduces the corresponding classical Poisson algebra. This is a sufficient condition to insure that the classical limit of the quantum theory that we are constructing reproduces the classical theory we started from. Since the correct classical limit is the sole requirement we have on the theory, the quantum diffeomorphism constraint defined in this way represents a consistent quantization of its classical counterpart.

2. We can use the transform, and define the loop representation vector constraint opererator as the transform of the vector constraint operator in the representation that diagonalizes $A$ and $\psi$.

3. We can express the classical vector constraint in terms of loop variables, as a suitable limit of a sequence of these variables. The corresponding quantum constraint is then defined as the limit of the corresponding quantum loop operators.

As in pure gravity, it is not difficult to show that these different strategies yeld the same quantum diffeomorphism constraint, and that this is can be expressed as follows. For every diffeomorphism $\phi \in \operatorname{Diff}[\Sigma]$

$$
\Psi[\alpha]=\Psi[\phi \cdot \alpha]
$$

where $[\phi \cdot \alpha](s):=\phi(\alpha(s))$. The general solution of the diffeomorphism constraint is the given by the loop functionals constant along the orbits of the action of the Diff group on the loop space, namely, they are given by

$$
\Psi[\alpha]=\Psi[K(\alpha)]
$$


where $K(\alpha)$ is a generalized knot class, that is, an equivalent class under diffeomorphisms of sets of graphs formed by open and closed lines.

For every generalized knot class $K$, we can define a corresponding quantum state $\Psi_{K}$ as the characteristic function of the class. We will also use a Dirac notation $\Psi[\alpha]=\langle\alpha \mid \Psi\rangle$, and denote the state $\Psi_{K}$ as $|K\rangle$. Thus

$$
\begin{aligned}
& \langle\alpha \mid K\rangle=1 \text { if } K=K(\alpha), \\
& \langle\alpha \mid K\rangle=0 \text { otherwise. }
\end{aligned}
$$

Let us begin here some preliminary investigation of the structure of the ensemble of quantum states $\mid K>$.

Consider a fixed class $K$. Let $\alpha$ be one of the (diff-equivalent) multiple loops that belongs to $K$. Let $\alpha$ be composed by $c$ closed loops and $o$ open loops. There are $2 o$ end points $\alpha_{e}$ in $\alpha$. We distinguish the end points as simple or doubles. An end-point $\alpha_{e}$ is simple is there is no other end point $\alpha_{e}^{\prime}$ in $\alpha$ such that $\alpha_{e}=\alpha_{e}^{\prime}$. It is double otherwise. Note that there may not be "triple" end points, because of the Pauli principle. Let $S$ and $D$ be the number of simple and double end points. And $N=S+2 D=2 o$ be the number of end points.

Consider the points $i$ in the immage of $\alpha$ such that one or more than one of the following is true:

1. $\alpha$ is non injective in $i$,

2. $i$ is an endpoint,

3. $\alpha$ is non differentiable in $i$;

we denote these points $i$ as generalised intersections, or, simply as intersections. We assume that the number of these intersections is finite, and we denote this number as $I$. Given an intersection $i$, we assume there is only a finite number of components of $\alpha$ coming out of it. We denote this number as $m_{i}$, and we call it the order of the intersection. Intersections of order 1 are single end points. Intersections of order 2 are either double end points or kinks along a loop. Intersections or order 3 are single end points that fall over a loop. Intersections of order 4 are either crossings of two loops, or a double endpoint that falls over a loop, and so on. We call the intersections of order one free end-points.

Consider an intersection $i$ of order $m_{i}$ in a loop $\alpha$. Let $\alpha_{j}(s)$, with $j=1 \ldots m_{i}$, and $\alpha_{j}(0)=i$, be the $m_{i}$ lines (components of the loop $\alpha$ ) that come out from $i$. Let $\vec{l}_{j}, \quad j=1 \ldots m_{i}$ be the $m_{i}$ tangents of $\alpha_{j}(s)$ in $i$. Let then $\vec{l}_{j}^{(k)}$, where $k$ is a positive integer, the $k$-th derivative of the $j$-th component of the loop in $i$. For instance, if $m_{i}=1$, and $i=\alpha(0)$, then $\vec{l}_{1}=d$ vecalpha $(s) / d s$, and $\vec{l}_{1}^{(2)}=d^{2} \vec{\alpha}(s) / d s^{2}$, and so on.

The vectors $\vec{l}_{j}^{(k)}$ transform among themselves under reparametrization of $\alpha$ and under diffeomorphisms. Consider the space of all possible intersections of 
order $m_{i}$. Consider to of these intersections as equivalent if they can be transformed into each other by diffeomorphisms or reparametrizations. Denote the space of the resulting equivalence classes as the moduli space of the intersection of order $m_{i}$. The moduli space of an intersection of order $n<5$ is discrete; not so, in general, for larger $n$. However, the moduli space is always finite dimensional. Let $a_{i}^{\left(m_{i}\right)}$ be a collection of parameters that coordinatizes the moduli space of the $i$ intersection, as well as characterizing the rootings of $\alpha$ through the $i$ intersection.

Finally, let $\mathcal{K}_{P}^{c}$ be a discrete index that labels the braids with $P$ (ordered) open hands and $c$ closed loops. We can then (over-) characterise a quantum state as (see ref. 河):

$$
\left|N, I, D ; \quad a_{1}^{m_{1}} \ldots . a_{I}^{m_{I}} ; \quad \mathcal{K}_{\sum_{i}{ }_{i} m_{i}}^{o}\right\rangle
$$

where, we recall, $N$ is the number of end-points, $I$ is the number of intersections, $D$ is the number of double end points, $m_{1} \ldots . m_{I}$ are the orders of the $I$ intersections, $a_{1}^{\left(m_{1}\right)} \ldots . a_{I}^{\left(m_{I}\right)}$ are the moduli space parameters of the intersections and $\mathcal{K}_{\sum_{i} m_{i}}^{o}$ is the discrete topological class of the braid obtained by deleting all intesection points from the loop. At the end of the paper we will describe some simple generalized knot classes and make use of this notation.

\section{The hamiltonian constraint has a simple ac- tion}

Our last and main task is to deal with the dynamics, which is contained in the hamiltonian constraint. (The Hamiltonian constraint of the pure gravity Loop Representation was discussed in 11, 12, 37]. For a comprehensive and critical discussion of the various approaches see ref. [38].) We shall perform this task in two stages. In the present section we introduce a simple and naive nonregularized definition of the quantum hamiltonian constraint. This is not really satisfactory because it does not allow us to control the divergences of the theory. However, we think it is usefull to present this non-regularized version of the dynamics first, because it allows one to appreciate the striking simplicity of the geometrical action of the Weyl-Einstein hamiltonain constraint, which otherwise could improperly appear as an improbable product of the technicalities of the regularization procedure. In the next two sections, we will transform the formal esxpressions we obtain here in a more solid result.

Thus, we begin by defining the hamiltonian constraint by using the simplest procedure: we define it in the connection representation and we transform it to the Loop Representation by using the Loop Transform [1]. We choose the ordering in which the momenta are always to the right of the configuration variables. Taking into account the Grassmann character of the fermionic variables, the 
Hamiltonian constraint, smeared against a test (inverse density) scalar $\underset{\sim}{N}(x)$ is

$$
\begin{aligned}
\widehat{H}[N]= & -\int_{\Sigma} d^{3} x \underset{\sim}{N} \operatorname{Tr}\left(F_{a b} \widehat{\widetilde{\sigma}}^{a} \widehat{\widetilde{\sigma}}^{b}\right)-i \sqrt{2} \widehat{\widetilde{\sigma}}_{A}^{a}{ }_{A} \mathcal{D}_{a} \psi^{A} \widehat{\widetilde{\pi}}_{B} \\
= & -\frac{1}{2} \int_{\Sigma} d^{3} x \underset{\sim}{N}(x) F_{a b B}^{A}(x) \frac{\delta}{\delta A_{a B}^{C}(x)} \frac{\delta}{\delta A_{b C}^{A}(x)} \\
& -i \mathcal{D}_{a} \psi^{A}(x) \frac{\delta}{\delta A_{b B}^{A}(x)} \frac{\delta}{\delta \psi^{B}(x)}
\end{aligned}
$$

In order to compute the Loop transform of this operator, we have to compute its action on the kernel of the Loop Transform, that is, on the basis loop states $\Psi_{\alpha}[A, \psi]$. We need to compute

$$
\widehat{H}[N] \Psi_{\alpha}[A, \psi] .
$$

If $\alpha$ is formed by closed loops alone, then $\Psi_{\alpha}[A, \psi]$ is independent from $\psi$ and therefore the second term in (56) does not act. It follows immediately that the action of $\widehat{H}[N]$ on the closed loop states is fully equivalent to the action of the pure gravity hamiltonian constraint. Let us then assume $\alpha$ is a single open loop. A straigth forward calculation gives

$$
\begin{aligned}
& \widehat{H}[N] \Psi_{\alpha}[A, \psi]= \\
& -\int_{0}^{1} d t \int_{0}^{1} d s \underset{\sim}{N}(\alpha(t)) \delta^{3}(\alpha(s), \alpha(t)) \dot{\alpha}^{a}(s) \dot{\alpha}^{b}(t) \\
& \psi^{G}\left(\alpha_{i}\right) U_{G[F}(0, t) F_{a b}{ }^{F B}(\alpha(t)) U_{B] H}(t, 1) \psi^{H}\left(\alpha_{f}\right) \\
& -\int_{0}^{1} d s \underset{\sim}{N}\left(\alpha_{i}\right) \delta^{3}\left(\alpha(s), \alpha_{i}\right) \dot{\alpha}^{a}(0) \mathcal{D}_{a} \psi^{A}\left(\alpha_{i}\right) U_{A E}[\alpha] \psi^{E}\left(\alpha_{f}\right) \\
& +\int_{0}^{1} d s \underset{\sim}{N}\left(\alpha_{f}\right) \delta^{3}\left(\alpha(s), \alpha_{f}\right) \dot{\alpha}^{a}(1) \psi^{D}\left(\alpha_{i}\right) U_{D A}[\alpha] \mathcal{D}_{a} \psi^{A}\left(\alpha_{f}\right) .
\end{aligned}
$$

We immediately see the difficulty in this equation: the three- dimensional delta functions are integrated only against two line integrals, leaving a divergent factor. As we said, let us disregard this infinity for the moment.

We now recall from ref.[1] that, if we disregard divergences, the action of the Hamiltonian operator on a pure loop state can be written as

$$
\widehat{H}[\stackrel{N}{\sim}] \Psi_{\alpha}=\mathcal{S}[\underset{\sim}{N}] \Psi_{\alpha}
$$

where $\mathcal{S}$, denoted as the "Shift operator" is a simple operator acting on the loop argument, as

$$
\mathcal{S}[\underset{\sim}{N}] \Psi_{\alpha}:=\int_{0}^{1} d s \int_{0}^{1} d t \underset{\sim}{N}(\alpha(s)) \dot{\alpha}^{a}(s) \delta^{3}(\alpha(s), \alpha(t)) \frac{\delta}{\delta \alpha^{a}(t)} \Psi_{\alpha} .
$$


If $\alpha$ has no self-intersections, and up to a divergent factor $k$, the Shift operator becomes simply

$$
\mathcal{S}[N] \Psi_{\alpha}=k \lim _{t->0} \Psi_{\alpha_{t N}}
$$

where

$$
\alpha_{t \underset{N}{\sim}}^{a}(s):=\alpha^{a}(s)+t \underset{\sim}{\sim}(\alpha(s)) \dot{\alpha}^{a}(s)
$$

The action of the Shift operator has thus a very simple geometrical interpretation: it shifts the loop ahead along its tangent. Clearly, it sends a smooth closed loop into itself, while it deforms a loop with kinks or intersections. This simple action is one of the ways in which one can (formally) understand the well known result that loop states corresponding to smooth non intersecting loops are in the kernel of the Hamiltonian constraint. The fact that the action of the hamiltonian constraint on the kernel of the Loop Transform can be expressed in terms of an operator acting on the loop variable enables us to interpret this operator as the operator that represents the Hamiltonian constraint in the Loop Representation [1]. Thus in pure gravity the Hamiltonian constraint can be simply expressed as the Shift operator.

How does the picture change if we include the fermions? The striking result that we have mentioned in the introduction is that the picture does not change at all with the introduction of fermions: Equations (49), which expresses the Hamiltonian as the Shift operator still holds. The shift operator is still given by equation $(50)$, where now we also allow the loops to be open, or in the absence of intersections, by equation (51). Indeed, by computing the action of $\mathcal{S}[N]$, as defined in equation (50) on an open loop state, we get precisely the right hand side of equation (48). What happens is that the fermion term in the classical hamiltonian constraint give rise to the second and third term in equation (48) and these terms are precisely the terms that "move" the two fermions at the end of the loop in the correct direction!

Thus we have the following result:

- In pure gravity the action of the hamiltonian constraint on Loop Space can be expressed as the action of the Shift operator (50), which simply shifts non-intersecting loops along their own tangent.

- By applying this same geometrical operator on open loop states, we have the action of the Weyl-Einstein hamiltonian constraint.

This result was first obtained by one of us in ref. [18]. The present paper represents an effort to clean up this result from the difficulties due to the divergent factor $k$.

We consider the fact that the coupled Einstein-Weyl equations can be coded in the extremely simple Shift operator as a striking fact. We have been deeply puzled by this result, and we do not see any simple way of interpreting it. We have not been able to find any reason for which this result could be understood in 
terms of the classical fermion dynamics. Its simplicity seems to us an indication of something, but we have not been able to decode the indication. We will return to this discussion in the conclusion section. For the moment, let us study how we can free the result from the divergences.

\section{QCD: dynamics}

\subsection{Coupling a clock field}

Following the ideas introduced in the introduction, we now modify our point of view, and consider a richer theory: We couple a scalar field to the Einstein-Weyl theory. Our aim to use the scalar field in order to define a physical internal time, or clock-time, in terms of which we can study physical evolution.

We have two independent motivations for chosing this roard. First this procedure allows us to unravel the physics of the general covariant quantum theory, otherwise hidden in the frozen-time formalism, as discussed in detail

for instance in refs. [9, 10]. Second, this is a way to overcome the divergence difficulties we had in the previous section. Indeed, we have learned from the experience in pure Quantum GR that non-diffeomorphism-invariant operators tend to be ill-defined in a generally covariant quantum field theory, while all the diffeomorphism invariant operators that we have been able to construct have good finiteness properties [2]. Thus, we wish to replace the Hamiltonian constraint with some diffeomorphism invariant operator [the Hamiltonian constraint, being a scalar (density) is diffeomorphism covariant, not diffeomorphism invariant]. As shown in ref.[B], the coupling of a scalar clock field and the replacement of the hamiltonian constraint with an hamiltonian is a way to acheive this result. The hamiltonian that generates the evolution in the clock time is a diffeomorphism invariant quantity and replaces the hamiltonian constraint.

We refer to [5] for the details of the scalar field construction and the gauge fixing that allows to define the hamiltonian. We simply recall here the main idea, so that this paper could be idependently read. Physical quantum states are represented (say in the connection representation) as functionals $\Psi[A, \psi]$ of the spacial fields $A(\vec{x}), \psi(\vec{x})$, satisfying the Wheler-DeWitt equation. As well known, the time coordinate $t$ disappears from this frozen time formalism. In theory, the disappearence of the coordinate time is not a problem, since the observables of the theory must be 4-dimensional general covariant anyway, and thus, in particular, must be independent from $t$ anyway. Examples of these 4-dimensional general covariant observables are given by the invariant distance $d_{p}$ of the solar system planets from the Earth, seen as a function dependent from, say, the invariant distance $d$ of the Earth from the sun, or as a function of the proper time (on the Earth) lapsed from some initial fixed event. These observables are manifestly coodinate invariant, and thus independent from $t$. In practice, however, it is notoriously too difficult to write the dynamical equa- 
tions of GR directly in terms of coordinate invariant quantities: in classical GR we almost always work with coordinate dependent quantities, and extract coordinate invariant predictions only after the dynamics has been fully worked out in a particular gauge. For instance, we study the motion of the planets, including perhaps emitted gravitational radiation, in some arbitrary coordinate system (with some arbitrary coordinate time $t$ : $d_{p}(t)$ and $d(t)$ ), and only when the dynamics has been solved we compute coordinate invariant quantities $d_{p}(d)$, which can be compared with astronomical observations. The quantum frozen time formalism, however, does not allow us to work with quantities dependent on the fictitious arbitrary coordinate time $t$, and thus makes the dynamical analysis particularly cumbersome: what one should do is to view the physical states $\Psi[A, \psi]$ as coding the quantum evolution of any variable in terms of any other variable. In general this is not easy.

The problem can be simplified by studying a version of the theory in which there is a simple quantity to be taken as the independent variable; that is, in which we know from scrath which variable we want to use as the "clock variable". We thus introduce a scalar field $T(\vec{x}, t)$, and we decide to study the evolution of the gravitational and fermions degrees of freedom, as they evolve in the value of $T$. If $A(\vec{x}, t), \psi(\vec{x}, t), T(\vec{x}, t)$ is a solution of the field equations, we extract information invariant under a time coordinate transformation by solving $t$ with respect to $T$, and substituting the resulting $t(\vec{x}, T)$ in $A$ and $\psi$ : We get the two functions

$$
\begin{aligned}
& A(\vec{x}, T)=A(\vec{x}, t(\vec{x}, T)), \\
& \psi(\vec{x}, T)=\psi(\vec{x}, t(\vec{x}, T)),
\end{aligned}
$$

which are invariant under coordinate time reparametrization. Equivaletly, we choose a (physical) coordinate system in which $T(\vec{x}, t)=t$. Of course, this cannot be done in general. It can only be done for certain solutions of the field equations, and for certain regions of spacetime. Thus, we are now beginning to use a formalism that holds only within a certain regime, and breaks down in approaching the limits of this regime - for instance, when the derivative $\frac{\partial T}{\partial t}$ vanishes.

In the quantum theory, the frozen time formalism is defined in terms of the functionals $\Psi[A, \psi, T]$ of the spacial fields $A(\vec{x}), \psi(\vec{x}), T(\vec{x})$. We can interpret these states as giving the amplitude for a $A(\vec{x}), \psi(\vec{x})$ configuration at the given configuration $T(\vec{x})$ of the clock field, and to interpret the Wheeler-DeWitt equation as an evolution equation in the multifingered time $T(\vec{x})$. We can then further fix the multifingered $T(\vec{x})$ time as a constant function $T(\vec{x})=T$, (we keep the same letter $T$ to indicate also the real number $T$, beside the function $T(\vec{x})$ ), and restrict $\Psi[A, \psi, T]$ (functional of the field $T(\vec{x})$ ) to $\Psi[A, \psi](T)=$ $\left.\Psi[A, \psi, T]\right|_{T(\vec{x})=T}$ (function of the real numeber $T$ ), without any loss of information. The state $\Psi[A, \psi](T)$ expresses the quantum amplitude for the evolution in $T$ of the fields $A(\vec{x}, T), \psi(\vec{x}, T)$ defined above. Moreover, if $\Psi[A, \psi, T]$ satsfies 
the Wheeler-DeWitt constraint, it is shown in $[5$ that $\Psi[A, \psi](T)$ satisfies the equation

$$
i \hbar \frac{\partial}{\partial T} \Psi[A, \psi](T)=\widehat{H} \Psi[A, \psi](T),
$$

where the operator $\widehat{H}$ will be defined in a moment. We can view equation (54) as a genuine Schrödinger equation which evolves in the time $T$.

More precisely, we can express the Einstein-Weyl-scalar-field theory in a canonical (gauge-fixed) form, in terms of the configuration variables $A_{a A}{ }^{B}$ and $\psi^{A}$, the gauge and diffeomorphisms constraints

$$
\begin{aligned}
\widetilde{G}_{A B} & =-i \sqrt{2} \mathcal{D}_{b} \widetilde{\sigma}^{b}{ }_{A B}+\widetilde{\pi}_{(A} \psi_{B)} \\
\widetilde{V}_{a} & =-i \sqrt{2} \operatorname{Tr}\left(\widetilde{\sigma}^{b} F_{a b}\right)-\widetilde{\pi}_{A} \mathcal{D}_{a} \psi^{A}
\end{aligned}
$$

and the hamiltonian (as opposed to hamiltonian constraint)

$$
H=\int d^{3} x \sqrt{-\operatorname{Tr}\left(\widetilde{\sigma}^{a} \widetilde{\sigma}^{b} F_{a b}\right)+i \sqrt{2} \widetilde{\sigma}_{A}^{a} \tilde{\pi}_{B} \mathcal{D}_{a} \psi^{A}} .
$$

It is shown in [5] that the solutions of this theory, $A(\vec{x}, T), \psi(\vec{x}, T)$, are related to the solutions of the full Einstein-Weyl-scalar-field theory via equation (53). We can define the kinematics of the quantum theory and treat the diffeomorphism constraint exactly as in the previous sections, but now we do not have a quantum hamiltonian constraint, but rather a Schödinger equation (54) and a quantum hamiltonian $\hat{H}$, which is the quantum operator corresponding to the classical variable $H$ given in (56).

The rest of this section is devoted to the construction of the quantum operator $\widehat{H}$. This time we will not be content with formal manipulations of divergent expressions, and we require a somehow higher level of rigor.

\subsection{Definition of the regularized hamiltonain}

In this section we derive the key techincal result of the paper, namely the explicit regularized action of the Hamiltonian on the knot classes; the section is quite long and technical - the final result is summarized in the following section 5.3. Following ref.[5], our first step is to definine a regularised classical hamiltonian in terms of loop variables. We introduce a fictitious background flat metric and a preferred set of coordinates in which this metric is euclidean; in the following everything is written in those coordinates and we use the euclidean metric to define norms of vectors and raise and lower vector indices. It will be our task, later, to show that the operator we define is independent from the regularization background metric introduced here. We write

$$
H=\lim _{\substack{L \rightarrow 0 \delta \rightarrow 0 \\ A \rightarrow 0} \rightarrow 0} H_{L \delta A \tau}
$$




$$
H_{L \delta A \tau}=\sum_{I} L^{3} \sqrt{-C_{\text {Einstein } I}^{A, L, \delta}-C_{\text {Weyl } I}^{L, \tau, \delta}} .
$$

Here we have partitioned three-dimensional space into cubes of sides $L$, labelled by the index $I$. The quantity $C_{\text {Einstein } I}^{A, L, \delta}$ is the regularized form of the pure gravity hamiltonian constraint (integrated over the $I$-th cube), and has been defined in 5 .

With the purpose of defining the fermion term $C_{\text {Weyl } I}^{L, \tau, \delta}$, we begin by defining the open loop $\gamma_{\vec{x}, \vec{y}}^{\tau}$, where $\tau$ is a regularization parameter (to be taken small), $\vec{x}$ is a point in space and $\vec{y}$ is a vector in the tangent space around $\vec{x}$. Since we have a background flat metric we can write expressions as $\vec{x}+s \vec{y}$, for any real $s$, with obvious meaning. The open loop $\gamma_{\vec{x}, \vec{y}}^{\tau}$ is defined as the (uniformly parametrized) straight line (in the background metric) that starts at $\vec{x}$ in the $\vec{y}$ direction and is long $\tau$. That is

$$
\left(\gamma_{\vec{x}, \vec{y}}^{\tau}\right)^{a}(0)=x^{a}, \quad\left(\dot{\gamma}_{\vec{x}, \vec{y}}^{\tau}\right)^{a}(s)=\frac{\tau}{|\vec{y}|} y^{a} .
$$

Note that

$$
\left(\gamma_{\vec{x}, \vec{y}}^{\tau}\right)^{a}(1)=x^{a}+\frac{\tau}{|\vec{y}|} y^{a}
$$

and

$$
\left(\gamma_{\vec{x}, \vec{y}}^{\tau}\right)^{a}(|\vec{y}| / \tau)=x^{a}+y^{a} .
$$

By making use of this loop, we define the regularised fermion hamiltonian constraint by

$$
\begin{aligned}
C_{\text {Weyl } I}^{L, \tau, \delta} & =\frac{1}{L^{3}} \int_{I} d^{3} x C_{\text {Weyl }}^{\tau, \delta}(\vec{x}) \\
C_{\text {Weyl }}^{\tau, \delta}(\vec{x}) & =c_{\tau \delta} \int d^{3} y \theta(\delta-|y|) \frac{y^{a}}{|\vec{y}|} Y^{a}\left[\gamma_{\vec{x} \vec{y}}^{\tau}\right](|y| / \tau) \\
c_{\tau \delta} & =\frac{3}{\tau} \frac{1}{\frac{4}{3} \pi \delta^{3}} .
\end{aligned}
$$

Here $\theta(x)$ is the conventional step function, that is the characteristic function of $R^{+}$. Note the three different roles of the three regularization parameters $L, \tau$ and $\delta$ : the parameter $L$ fixes the size of the boxes. As we will see, the introduction of these boxes will allow us to deal with the square root. For every space point $x$, the fermion term of the Hamiltonian constraint $C_{\text {Weyl }}(\vec{x})$ is approximated by means of the loop variable $Y^{a}$ corresponding to the "small" loop $\gamma_{\vec{x} \vec{y}}^{\tau}$, that starts at $x$. The parameter $\tau$ gives the length of the "small" loop. The direction of this loop is integrated over ( $d^{3} y$ angular integration). $Y^{a}$ has a special point where the hand is inserted. This point is chosen to be $\gamma_{\vec{x} \vec{y}}^{\tau}(|y| / \tau)=x^{a}+y^{a}$. Thus, also the position of this point (the hand) is integrated over $\left(d^{3} y\right.$ radial integration). This smearing of the position of the 
hand is what determines the point split of the functional derivatives operators in the quantum theory. Note that the $d^{3} y$ integral is restricted (by the $\theta$ function) to a ball of size $\delta$ around $x$. Thus, the parameter $\delta$ gives the point splitting separation between the initial point of the loop and its hand. Note that in order this definition to make sense we must have $\tau>\delta$.

A straigthforward expansion in $\tau$ and $\delta$ shows that equation (57) is satisfied, namely that the quantity that we have defined represents a genuine regularization of the Hamiltonian. For completness, let us sketch this expansion. We begin by writing the regularized expression explicitely, by using the expression for $Y^{a}$, given in eq.(29)

$$
\begin{aligned}
C_{\text {Weyl }}^{\tau, \delta}(\vec{x})= & c_{\tau \delta} \int d^{3} y \theta(\delta-|y|) \frac{y^{a}}{|\vec{y}|} \pi^{A}\left(\gamma_{\vec{x} \vec{y} i}^{\tau}\right) U_{A}{ }^{B}\left[\gamma_{\vec{x} \vec{y}}^{\tau}\right](0,|y| / \tau) \\
& \tilde{\sigma}^{a}{ }_{B}{ }^{C}\left(\gamma_{\vec{x} \vec{y}}^{\tau}(|y| / \tau)\right) U_{C}{ }^{D}\left[\gamma_{\vec{x} \vec{y}}^{\tau}\right](|y| / \tau, 1) \psi_{D}\left(\gamma_{\vec{x} \vec{y} f}^{\tau}\right) .
\end{aligned}
$$

By using the explicit definition of the loop $\gamma_{\vec{x} \vec{y}}^{\tau}$, this becomes

$$
\begin{aligned}
C_{\text {Weyl }}^{\tau, \delta}(\vec{x})= & c_{\tau \delta} \int d^{3} y \theta(\delta-|y|) \frac{y^{a}}{|\vec{y}|} \pi^{A}(\vec{x}) U_{A}{ }^{B}\left[\gamma_{\vec{x} \vec{y}}^{\tau}\right](0,|y| / \tau) \\
& \tilde{\sigma}^{a}{ }_{B}^{C}(\vec{x}+\vec{y}) U_{C}{ }^{D}\left[\gamma_{\vec{x} \vec{y}}^{\tau}\right](|y| / \tau, 1) \psi_{D}(\vec{x}+\tau \vec{y} /|\vec{y}|) .
\end{aligned}
$$

We may now expand everything in powers of $\delta$ and $\tau$ around the point $\vec{x}$. Before doing so, however, let us note what follows. Since we have a $\left(d^{3} y\right)$ integration over a volume of order $\delta^{3}$, and we divide by $\delta^{3}$ (in $c_{\tau \delta}$ ) before taking the limit $\delta \rightarrow 0$, only the term in the integrand of zero order in $\delta$ may survive the limit. We also divide by $\tau$ and take the limit $\tau \rightarrow 0$, thus only terms of first order in $\tau$ survive in the limit. This means that to the relevant order we may replace quantities in $\vec{x}+\vec{y}$ by quantities in $\vec{x}$ (recall the $d^{3} y$ integration is over a sphere of radius $\delta$ ), and quantities in $\vec{x}+\tau \vec{y} /|\vec{y}|$, which is a distance $\tau$ from $\vec{x}$ by the first two terms in their Taylor expansion around $\vec{x}$. By doing so, the first of the two parallel propagators is just replaced by the identity, while the second can be replaced with the entire parallel propagator along the small loop. We therefore have, up to terms of order $\delta$ or $\tau$,

$$
\begin{aligned}
C_{\text {Weyl }}^{\tau, \delta}(\vec{x})= & c_{\tau \delta} \int d^{3} y \theta(\delta-|y|) \frac{y^{a}}{|\vec{y}|} \pi^{A}(\vec{x}) \delta_{A}{ }^{B} \\
& \tilde{\sigma}^{a}{ }_{B}{ }^{C}(\vec{x})\left(\delta_{C}{ }^{D}+\tau y^{c} A_{c C}^{D}(\vec{x})\right)\left(\psi_{D}(\vec{x})+\tau y^{c} \partial_{c} \psi_{D}(\vec{x})\right)= \\
= & c_{\tau \delta} \int d^{3} y \theta(\delta-|y|) \frac{y^{a}}{|\vec{y}|} \pi^{A}(\vec{x}) \\
& \tilde{\sigma}^{a}{ }_{A}{ }^{C}(\vec{x})\left[\psi_{C}(\vec{x})+\tau y^{c}\left(A_{c C}^{D}(\vec{x}) \psi_{D}(\vec{x})+\partial_{c} \psi_{C}(\vec{x})\right)\right]
\end{aligned}
$$

The first term in the square brackets vanishes because $\int d^{3} y y^{a}=0$, and the second term is the covariant derivative. Thus

$$
C_{\text {Weyl }}^{\tau, \delta}(\vec{x})=c_{\tau \delta} \tau\left[\int d^{3} y \theta(\delta-|y|) \frac{y^{a} \vec{y}^{c}}{|\vec{y}|}\right] \quad \pi^{A}(\vec{x}) \tilde{\sigma}^{a}{ }_{A}^{C}(\vec{x}) D_{c} \psi_{C}(\vec{x}) .
$$


The integration is now immediate

$$
\int d^{3} y \theta(\delta-|y|) \frac{y^{a} y^{c}}{|\vec{y}|}=3 \delta^{a c} \frac{4}{3} \pi \delta^{3}
$$

Restoring the explicit form of $c_{\tau \delta}$ we thus obtain

$$
C_{\text {Weyl }}^{\tau, \delta}=\pi^{A} \tilde{\sigma}_{A}^{a}{ }^{C} D_{c} \psi_{C}+O(\delta)+O(\tau),
$$

namely the fermion term of the hamiltonian constraint, as we wanted.

We have shown that the quantity (63) provides indeed a regularized form of the fermion hamiltonian constraint. We now come to the quantum theory and define the corresponding regularized quantum operator

$$
\begin{aligned}
\widehat{H}_{L \delta A \tau} & =\sum_{I} L^{3} \sqrt{-\hat{C}_{\text {Einstein } I}^{A, L, \delta}-\hat{C}_{\text {Weyl } I}^{L, \tau, \delta}} . \\
\hat{C}_{\text {Weyl } I}^{L, \tau, \delta} & =\frac{1}{L^{3}} \int_{I} d^{3} y \hat{C}_{\text {Weyl }}^{\tau, \delta}(y), \\
\hat{C}_{\text {Weyl }}^{\tau, \delta}(y) & =c_{\tau \delta} \int d^{3} x \theta(\delta-|y|) \frac{y^{a}}{|y|} \hat{Y}^{a}\left[\gamma_{\vec{x} \vec{y}}^{\tau}\right](|y| / \tau) .
\end{aligned}
$$

The operator $\hat{Y}^{a}$ is defined in equation (41). Note that in this regularized operator the two hands of the operator [1] do not overlap: they are point split.

Let us study the action of the operator that we have defined: in spite of it apparent complexity, this action will turn out to be relatively simple. Plugging the explicit definition of $\widehat{Y}^{a}$ (41) in (73) gives

$$
\begin{aligned}
\hat{C}_{\text {Weyl }}^{L, \tau, \delta}(\vec{x}) \Psi[\alpha]= & c_{\tau \delta} \int d^{3} y \theta(\delta-|y|) \frac{y^{a}}{|y|} \sum_{\alpha_{e}} \delta^{3}\left(\vec{x}, \alpha_{e}\right) \\
& \int_{\alpha} d s \dot{\alpha}^{a}(s) \delta^{3}\left(\gamma_{\vec{x} \vec{y}}^{\tau}(|\vec{y}| / \tau), \alpha(s)\right) \sum_{q= \pm} \Psi\left[\alpha * *^{q}{ }_{e, s} \gamma_{x y}^{\tau}\right](7.4)
\end{aligned}
$$

We now use the explicit form of the small loop, and we keep only terms small in $\tau$ and $\delta$. The first $\delta^{3}$ function in the last equation forces the loop $\alpha$ to have an end-point $\alpha_{e}$ in $\vec{x}$. The integration over the small ball (size $\delta$ ) around this point, and the second $\delta^{3}$ function, pick up a second point $\alpha(s)=\vec{x}+\vec{y}$ in $\alpha$, close to $\alpha_{e}$. If $\alpha_{e}$ is a free end point, we can write this second point (up to the relevant order) as $\vec{x}+|y| \vec{l}_{e}$, where $\vec{l}_{e}$ was defined in the previous section as the tangent of $\alpha$ in the end-point $\alpha_{e}$. In general, however, $\alpha_{e}$ needs not be a free end-point; in the general case there will be several components of $\alpha$ originating from $\alpha_{e}$. Thus $\vec{l}_{e}$ takes a finite number of values, and the radial $d^{3} y$ integration together with radial part of the second delta function pick up all these values; note that they turn out to be proportional to $\vec{y}$. The radial $d^{3} y$ integration is then straightforward, and we obtain

$$
\hat{C}_{\text {Weyl }}^{L, \tau, \delta}(\vec{x}) \Psi[\alpha]=\frac{c_{\tau \delta}}{L^{3}} \delta \sum_{\alpha_{e}} \delta^{3}\left(\alpha_{e}, \vec{x}\right) \sum_{\vec{l}_{e}} \sum_{q= \pm} \Psi\left[\left[\alpha * *^{q}{ }_{e, \delta} \gamma_{\vec{x}(\vec{x}+\delta \vec{l})}^{\tau}\right] .\right.
$$


We introduce the notation

$$
\begin{gathered}
\hat{C}_{\text {Weyl }}^{L, \tau, \delta}(x) \Psi[\alpha]=\frac{c_{\tau \delta}}{L^{3}} \delta \sum_{\alpha_{e}} \delta^{3}\left(\alpha_{e}, x\right) \widehat{\mathcal{F}}_{e}^{\tau \delta} \Psi[\alpha] \\
\widehat{\mathcal{F}}_{e}^{\tau \delta} \Psi[\alpha]=\sum_{\vec{l}_{e}} \sum_{q= \pm} \Psi\left[\left[\alpha * *^{q}{ }_{e, \delta} \gamma_{\vec{x}(\vec{x}+\delta \vec{l})}^{\tau}\right] .\right.
\end{gathered}
$$

Finally, we may come to the Hamiltonian. Let us assume for a moment that the loop we are dealing with has no intersection nor kinks, so that we can set the Einstein term to zero. Inserting our last result into the Hamiltonian we have

$$
\hat{H} \Psi[\alpha]=\lim _{\substack{L \rightarrow 0 \delta \rightarrow 0 \\ \tau \rightarrow 0}} \sum_{I} L^{3} \sqrt{\frac{c_{\tau \delta}}{L^{3}} \delta \int_{\text {cube } I} d^{3} x \sum_{\alpha_{e}} \delta^{3}\left(\alpha_{e}, x\right) \widehat{\mathcal{F}}_{e}^{\tau \delta}} \Psi[\alpha] .
$$

For $L$ small enough, and assuming that $\delta<<L$, so that "boundary effects" of the box can be neglected, every cube $I$ contains only one end-point, and since $\hat{C}_{\text {Weyl }}$ gives zero unless there is an end-point in $I$, we have

$$
\hat{H} \Psi[\alpha]=\lim _{\substack{\tau \rightarrow 0 \delta \rightarrow 0 \\ \tau \rightarrow 0}} \sqrt{\frac{3}{\tau} \frac{1}{\frac{4}{3} \pi \delta^{3}} L^{3} \delta} \sum_{\alpha_{e}}\left(\widehat{\mathcal{F}}_{e}^{\tau \delta}\right)^{-\frac{1}{2}} \Psi[\alpha] .
$$

where we have restored the explicit expression for $c_{\tau \delta}$.

It is now time to study the limits explicitely. Let us first focuss on the crucial prefactor

$$
C(L, \tau, \delta)=\sqrt{\frac{3}{\tau} \frac{1}{\frac{4}{3} \pi \delta^{3}} L^{3} \delta}=\frac{3}{2 \sqrt{\pi}} \sqrt{\frac{L^{3}}{\tau \delta^{2}}} .
$$

The question we have to address is the finitness of the above limit. Clearly the result depends on the order in which the limits are taken. This is precisely what we should have expected: different orders in which the limit is taken correspond to inequivalent definitions of the quantum operator. Since all these definitions correspond to the same classical limit, the choice amount to a choice of different orderings of the quantum hamiltonian. The question is whether there is one choice that gives us a finite quantum operator.

Of course we may not confine ourselves to the choice between taking one first or another one first of the three limits: we choose any combination. More precisely, we may consider the three dimensional $L, \tau, \delta$ space, and study the limit of $C(L, \tau, \delta)$ as we approach the point $L=0, \tau=0, \delta=0$ : this point can be approached in a variety of alternative ways, not just along one of the coordinate axis. Let us introduce a parameter $\epsilon$, and consider a curve $L(\epsilon), \tau(\epsilon), \delta(\epsilon)$ in this tree dimensional space, such that $L(0)=0, \tau(0)=0, \delta(0)=0$. Our problem is to understand wether we can choose this curve in such a way that the limit

$$
\lim _{\epsilon \rightarrow 0} C(L(\epsilon), \tau(\epsilon), \delta(\epsilon))
$$


is finite. We are not free to choose the curve $L(\epsilon), \tau(\epsilon), \delta(\epsilon)$ in a completly arbitrary way, because there is a certain number of conditions that we have imposed on the regularization parameters along the way. First, of course, we must have $L>0, \tau>0, \delta>0$. Then, have required $\tau>\delta$, and, in order to avoid "boundary effects" in the box, $L>\delta$. Can all the conditions be satisfied and a finite limit be obtained ?

The first crucial point to be noted is that powers of lengths cancell exactly and the quantity $C(L, \tau, \delta)$ is dimensionless. This is a necessary condition for having a finite limit. (It was precisely the fact that we got a divergent quantity with the dimensions of a length, measured in the background metric, that prevented us from achieving a background independent renormalization in section (5)). By itself, however, this fact does not suffice to garantee a well defined limit. We now claim that this limit can indeed be chosen consistently with all the demands, as follows

$$
\begin{aligned}
L(\epsilon) & =k \epsilon^{3} a, \\
\tau(\epsilon) & =\epsilon a, \\
\delta(\epsilon) & =\epsilon^{4} a,
\end{aligned}
$$

where $a$ is an arbitrary length, and $k$ is a arbitrary dimensionless positive number. It is easy to see that all the requested conditions are satisfied. In the limit, we have

$$
\lim _{\epsilon \rightarrow 0} C(L(\epsilon), \tau(\epsilon), \delta(\epsilon))=\frac{3}{2 \sqrt{\pi}} \sqrt{\frac{L^{3}(\epsilon)}{\tau(\epsilon) \delta^{2}(\epsilon)}}=\frac{3 k^{-3 / 2}}{2 \sqrt{\pi}}:=\lambda^{2}
$$

$\lambda$ is a free dimensionless constant that emerges from the regularization procedure. Thus, the prefactor is finite in the limit.

Thus, we write the action of the hamiltonian as

$$
\hat{H} \Psi[\alpha]=\lambda^{2} \quad \sum_{\alpha_{e}}\left(\widehat{\mathcal{F}}_{e}\right)^{-\frac{1}{2}} \Psi[\alpha]
$$

where we have introduced the "end-point operator"

$$
\widehat{\mathcal{F}}_{e} \Psi[\alpha]=\lim _{\epsilon \rightarrow 0} \widehat{\mathcal{F}}_{e}^{\tau(\epsilon) \delta(\epsilon)} \Psi[\alpha] .
$$

We now examine this "end-point operator", its action, its finiteness and its transformation properties under diffeomorphisms.

Since $\delta$ (the point splitting distance of the two grasps) goes to zero much faster than $\tau$ (the length of the added loop, we can now simply take the $\delta \rightarrow 0$ limit first, and the $\tau \rightarrow 0$ limit second. Let us consider the $\delta \rightarrow 0$ limit of $\widehat{\mathcal{F}}_{e}^{\tau \delta} \Psi[\alpha]$ (with finite $\tau$ ). It is easy to see that if the end- point is free, the action of the operator is simply to add a small straight line of length $\tau=\epsilon a$ to the end point of the loop, in the direction of the loop tangent (goign out from the 
intersection). If the end-point is not free, the action of the operator produces one term for each component of $\alpha$ emerging from the end-point. The terms corresponding to the component of $\alpha$ that ends in $\alpha_{e}$ is again just an addition of a small straight line to the end-point, emerging from the end point in the same direction as the original loop; while the other terms imply the addition of the small loop and also a rerouting through the intersection. The rerouting pattern can be calculated in straight forward way from equation (77).

Before taking the limit $\tau \rightarrow 0$, let us now assume that $\Psi[\alpha]$ is a diffeomorphism invariant state. Thus $\Psi[\alpha]$ depends on the diffeomorphism equivalence class of $\alpha$ only. If $\alpha_{e}$ is a free end-point, we have then

$$
\lim _{\tau \rightarrow 0} \widehat{\mathcal{F}}_{e}^{\tau 0} \Psi[\alpha]=\lim _{\tau \rightarrow 0} 2 \Psi\left[\alpha * * \gamma_{\alpha_{e},\left.\dot{\alpha}\right|_{e}}^{\tau}\right]=2 \Psi[\alpha],
$$

because for small enough $\tau$ the added loop will not interject any other loop, and the addition of a small line at the end of a loop does not change the diffeomorphism equivalence class of the loop.

If, on the other way, $\alpha_{e}$ is not a free end-point, then the loop $\alpha * * \gamma_{\alpha_{e}, \vec{l}_{e}}^{\tau}$ does belong to a different knot class than $\alpha$. For instance, assume the end point $\alpha_{e}$ falls over a smooth component $\beta$ of the loop $\alpha$. One of the terms in (77) adds a small straight line to $\alpha$. SInce this in the direction in which $\alpha$ emerges from the intersection, its action is essentially to cut away an infinitesimal portion of the loop at the end point. This can be seen by noticing that we can assume without loosing generality that $\alpha$ is a straight line at the intersection, and using the retracing identity, to "retrace" $\alpha$ away from the intersection along the small added loop. In the limit, we go back to $\alpha$; however, for every finite value of the regularization parameter, the knot class has changed: we are in the knot class in which $\alpha$ does not touch $\beta$. Thus, by continuity in the limit we still have the knot class in which $\alpha$ does not touch $\beta$. The key point, now, is that in any case, since $\Psi[\alpha]$ is diffeomorphism invariant, for small enough $\tau$ we have that $\widehat{\mathcal{F}}_{e}^{\tau 0} \Psi[\alpha]$ becomes independendent from $\tau$. Therefore the limit is the limit of a constant function, and therefore is finite. The action of the Hamiltonian, therefore, has opened up the intersection between $\beta$ and the end point of $\alpha$.

It is clear that the resulting action of $\widehat{\mathcal{F}}_{e}$ is well-defined on the diffeomorphism invariant states. Thus, the operator $\widehat{H}$ is finite and diffeomorphism invariant in the limit.

If we now reinstate $\hat{C}_{\text {Eintsein }} \neq 0$, we have

$$
\hat{H}=\sum_{\substack{\text { intersections } i \\ \text { end-points } e}} \sqrt{\hat{M}_{i}+\lambda \hat{\mathcal{F}}_{e}},
$$

where $\hat{M}$ was constructed in [5]. $\hat{H}$ is a finite operator defined on knot states. Its action follows immediately from the construction above. 


\subsection{Topological Feynman rules}

It is clear from the discussion above that the action of the Hamiltonian constraint on the generalized knot classes can be represented in a fully geometrical way in terms of the action of the operator on single intersections. The operator "opens up" intersections, and rearrange the rootings through the intersections. The details of this action can be computed from equations (778590), which we report here for easy reference. The finite geometrical action on the loops is defined by the operator 77 )

$$
\widehat{\mathcal{F}}_{e}^{\tau \delta} \Psi[\alpha]=\sum_{\vec{l}_{e}} \sum_{q= \pm} \Psi\left[\left[\alpha * *^{q}{ }_{e, \delta} \gamma_{\vec{x}(\vec{x}+\delta \vec{l})}^{\tau}\right] .\right.
$$

This operator attaches to every intersection and to every line coming out from an intersection two small straight loop, one for each one of the two possible rearranging of the rootings at the intersection between the added little loop and the line. Next, we take the limit (85)

$$
\widehat{\mathcal{F}}_{e} \Psi[\alpha]=\lim _{\epsilon \rightarrow 0} \widehat{\mathcal{F}}_{e}^{\tau(\epsilon) \delta(\epsilon)} \Psi[\alpha] .
$$

And finally, the Hamiltonian is given by 90

$$
\hat{H}=\sum_{\substack{\text { intersections } i \\ \text { end-points } e}} \sqrt{\hat{M}_{i}+\lambda \hat{\mathcal{F}}_{e}} .
$$

The matrix elements of the operators $\hat{M}_{i}(\sqrt[5]{)})$ and $\hat{F}_{e}$ can be directly computed between any two given knot states. The calculation amounts in a straightforward exercize in geometry and combinatorics, starting from the two equations above. The next problem is to compute the square root of the resulting (infinite) matrix. We expect that the square root can be computed order by order as the complexity of the knots considered increase. Work is in progress to compute explicitely the matrix elements, and thus understand if the structure of the resulting matrix allows a simple argoritm for extracting the square root. The resulting geometrical action represents the equivalent of the Feynman rules for this diffeomorphism invariant, or (infinite dimensional) topological, theory. We suggest to denote them as topological Feynman rules.

\section{QGD: dynamics}

We are now in the position of describing the general structure of Quantum Gravitational Dynamics, or QGD, the quantum theory of gravitationally interacting fermions, evolving in the clock time defined by a scalar field.

A physical quantum state $|K\rangle$ of the theory is specified by a generalized knot, namely an open braid $K$ of order $N$ (with $N$ open end-points, $N$ even), 
with an arbitrary finite number $I$ of intersections. A more accurate notation for these states is given in equation (45). The quantum dynamics is given by the matrix $\hat{H}$ in braid space, given in equation (90), the matrix elements of which are computed, order by order, according to the geometrical and algebraic rules given by equations (77) and (85), and in ref. 55 . We can interpret the matrix elements of $\hat{H}$ as first order transitions amplitudes in a time dependent perturbation expansion in the clock time $T$. In principle, the exponentiation of the $\hat{H}$ action gives the full evolution.

\subsection{The simplest states}

For instance, we can start from the simplest state formed by a single nonself intersecting open line. In terms of the notation (45), this can be denoted as

$$
\left|2,2,0 ; 1_{2}^{0}\right\rangle
$$

where we have indicated the simplest value of $\mathcal{K}_{2}^{0}$, a single line, by $1_{2}^{0}$. The moduli space of free open-ends is clearly formed by a single point, and thus we do not need $a_{i}$ parameters.

There are two fermions in this quantum state. We have that $\hat{M}_{i}\left|2,2,0 ; 1_{2}^{0}\right\rangle=$ 0 because there are no intersections of kinks in $\left|2,2,0 ; 1_{2}^{0}\right\rangle$. On the other side, we have from (86) that

$$
\hat{F}_{e}\left|2,2,0 ; 1_{2}^{0}\right\rangle=\lambda^{2}\left|2,2,0 ; 1_{2}^{0}\right\rangle
$$

so that we get

$$
\hat{H}\left|2,2,0 ; 1_{2}^{0}\right\rangle=2 \lambda\left|2,2,0 ; 1_{2}^{0}\right\rangle .
$$

Therefore $\left|2,2,0 ; 1_{2}^{0}\right\rangle$ is an eigenstate of the theory, or equivalently, the time dependent Schrödinger quantum state

$$
\left|2,2,0 ; 1_{2}^{0}, \quad T\right\rangle=\exp \left\{i \lambda \sqrt{\frac{c^{5}}{\hbar G}} T\right\}\left|2,2,0 ; 1_{2}^{0}\right\rangle
$$

is a solution of the exact quantum interacting theory. (We have restored physical units, for clarity.) Perhaps this state corresponds to an extremely simple "universe" in which there are only two fermions gravitating around each other in the simplest of the quantum geometries. It is suggestive to think at this state as a kind of "atomic" "ground state" of a simple 2-fermions universe.

Next we can consider the generalized knot formed by $n$ non intersecting copies of the above, and denote it as $\left|2 n, 2 n, 0 ; 1_{2 n}^{0}\right\rangle$. It is then straight forward to see that the time evolution of this state is given by

$$
\left|2 n, 2 n, 0 ; 1_{2 n}^{0}, \quad T\right\rangle=\exp \left\{i n \lambda \sqrt{\frac{c^{5}}{\hbar G}} T\right\}\left|2 n, 2 n, 0 ; 1_{2 n}^{0}\right\rangle
$$


and that the corresponding energy eigenstates are

$$
E_{n}=n E_{1}=n \lambda \sqrt{\frac{c^{5} \hbar}{G}} .
$$

As soon as we consider simple intersecting states, the full complexity of the operators $\hat{M}_{i}$ and $\hat{F}_{e}$ becomes relevant, and we have non-trivial time evolution.

\subsection{Comments}

Before concluding, we list here a certain number of comments and considerations, as well as pointing out several important problems that remain open.

1. Conservation of particle number. The operator $\hat{F}_{e}$ defined above acts on end-points by displacing them, and possibly by changing the associated rootings at intersections, but never creates or destroys end-points. Since the operator $\hat{M}_{i}$ too, conserves the number of end-points, it follows that the hamiltonian that we have defined conserves the number of particles. This is at first surprising, given that in general there is particle creation from space-time dynamics. But a more close analysis shows that this conservation is to be expected. Unlikely the Einstein-Dirac theory, indeed, the Einstein-Weyl theory does conserves particle number. This can be seen classically from the fact that the quantity

$$
N:=\int_{\Sigma} d^{3} x \psi^{A}(x) \widetilde{\pi}_{A}(x)
$$

commutes with all the constraints, including the hamiltonain constraint 30. One can immediately define the corresponding operator (say, using the Loop Transform), which turns out to be

$\left.\left.\hat{N} \| N, I, D ; \quad a_{1}^{m_{1}} \ldots . a_{I}^{m_{I}} ; \quad \mathcal{K}_{\sum_{i} m_{i}}^{o}\right\rangle=N \| N, I, D ; \quad a_{1}^{m_{1}} \ldots . a_{I}^{m_{I}} ; \quad \mathcal{K}_{\sum_{i} m_{i}}^{o}\right\rangle$.

This confirms the interpretation of the number $N$ of end-points as the particle number. Since $[\hat{N}, \hat{H}]=0$, the number of end-points $N$ is a conserved quantum number in the theory.

2. Particle anti-particle distinction. The Weyl field theory describe a particleantiparticle couple (say a neutrino and its anti- neutrino). In the Langrangian formulation the fermions are described by two complex fields. Since the action contains only first derivatives, the phase space has the same dimension as the space of the lagrangian fields, namely four real dimensions per point. These give two degrees of freedom, which describe, indeed, the particle and its antiparticle. Do the end-points of the loops represent particles or anti-particles? The answer is that the distinction 
is not gauge invariant, thus the question is not well posed in the theory. In flat space one can globally distinguish particles from antiparticles; but when the Weyl system is coupled with gravitation, something curious happens: the particle antiparticle distinction becomes local. Consider two field excitations in two different space position, and assume the first is a particle; then, the fact that the second be a particle or an antiparticle depends on the parallel transport operator between the two. This is because the particle and the antiparticle are distinguished by different directions in the internal spin space, and we can only compare directions in spin space in different points by using the connection. Since the particle anti-particle distinction is gauge dependent, in a gauge-independent formulation there is not way to distinguish particles from anti-particles. This is why the end-points of the loop represent at the same time both kinds of excitations.

3. Regime of validity of the formalism, and complex energy eigenvalues. This is an important feature of the clock field formalism that we must be discussed in detail. The formalism cannot be used in any regime of the system. This is already obvious at the classical level: Consider an arbitrary solution of the field equations $A(\vec{x}, t), \psi(\vec{x}, t), T(\vec{x}, t)$ : in general it is not possible to invert $T(\vec{x}, t) \rightarrow t(\vec{x}, T)$ globally. Thus, we certainly cannot use $T$ as a time variable for every solutions of the field equations and for every spacetime region. On the other side, there are solutions and spacetime reions where we can make the inversion. Consider an initial configuration of the $A(\vec{x}), \psi(\vec{x}), T(\vec{x})$ fields and their time derivatives $\dot{A}(\vec{x}), \dot{\psi}(\vec{x}), \dot{T}(\vec{x})$ on a given space like surface. This defines a point in phase space. Assume that $\dot{T}(\vec{x})<0$ in a spacial region $R$. There will be a time interval $\Delta t$ for which $\dot{T}(\vec{x})$ will remain positive in $R$. More precisely, we can determine a region $\Gamma$ in phase space, and a corresponding spacetime region $S$, such that for any initial condition in $\Gamma, \dot{T}(\vec{x})$ is positive in $S$. We shall say that the gravitational-fermion-scalar field system is in the clock regime in $S$ if the initial conditions are in $\Gamma$, namely if $\dot{T}(\vec{x})$ is positive all over $S$. By definition, we can perform the inversion $T(\vec{x}, t) \rightarrow t(\vec{x}, T)$ in the region in which the system is in the clock regime. Thus, as far as the classical theory is concerned, the formalism makes sense only in this regime. The same is true in the quantum theory. The quantum formalism that we have constructed is meanigfull in the clock regime.

A paradigm for this construction can be found in the quantum system of two uncoupled simple harmonic oscillator variables $g(t), f(t)$, if we fix the total energy $E$ and decide to never consider the evolution in the external clock time $t$, but rather use one of the two variables, say $f$ as internal clock; namely if we decide to ask questions concerning what is the position $g$ of the first oscillator, when the second is in $f$. We obtain the classical evolution $g(f)$ by inverting $f(t) \rightarrow t(f)$ and defining $g(f)=g(t(f))$. 
We can also do the same in quantum mechanics (see ref. [21], where the example is worked out in detail). However, along any orbit there is a point in which the internal time variable $f$ "comes back" (in $t$ ), and therefore we obtain a non unitary evolution operator in $f$. The physical interpretation of this non-unitarity is clear: there is "no system" anymore for $f$ arbitarry large.

The formalism reflect this fact, both classically and quantum mechanically, in the form of the hamiltonian. The hamiltonian that evolves the two oscillators in the external time $t$ is $p_{g}^{2}+p_{f}^{2}+g^{2}+f^{2}$. The hamiltonian that evolves the systemin the internal time $f$ is easilly obtained solving for $p_{f}$ (see ref. 21])

$$
H(f)=\sqrt{E-p_{g}^{2}-g^{2}-f^{2}}
$$

(where $E$ is the total energy of the system in the $t$ time). This (time dependent) hamiltonian generates the evolution equations for $g(f)$. The important point to note is that the hamiltonian becomes immaginary for large $f$. This simply signal the fact that it doesn't make sense anymore to evolve $g(f)$ in $f$. If we want to continue the evolution by using an internal time, we have to choose another, distinct, internal time, and "patch" the evolution. Note that this does not mean that the formalism that evolves in $f$ is inconsistent or incorrect: it simply means that is has a certain domain of validity. The same holds in quantum mechanics. Indeed, it was shown in ref. 21] that the quantum hamiltonian corresponding to (99) is self- adjoint when suitably restricted to an ( $f$-dependent) region of the Hilbert space, but develops immaginary eigenvalues if applied outside this region.

Similarly, we expect that the the hamiltonian that we have defined will also have immaginary eigenvalues. These simply signal that twe are going out from the domain of validity of the formalism, namely from the clock regime. We are trying to use evolution in $T$ to describe the gravitational field in regions where the $T$ fields fails to be monotonic. Explicitely, this possibility can be easilly traced back to the classical hamiltonian constraint. Roughly speaking, since the form of this constraint is $\Pi^{2}+C_{\text {Einstein }}+C_{\text {Weyl }}=0$ ( $\Pi$ being the scalar field momentum) and since in order $\dot{T}$ to change sign $\Pi$ must vanish, it follows that the save region, is where $C_{\text {Einstein }}+C_{\text {Weyl }}>0$, which is of course a sufficient condition for the Hamiltonian we have definined, $H=\int \sqrt{C_{\text {Einstein }}+C_{\text {Weyl }}}$ to be real. Thus, imaginary eigenvalues of $\hat{H}$ signal that we are exiting the regime of validity of the formalism we have developed here: the object we have chosen as clock is running backward. We must therefore exclude from the state space of QGD, as formulated here, the graphs that are eigenvalues of $\hat{H}$ witha an eigenvalue that is not a real positive number. 
In particular, all the vacuum solutions of pure quantum GR that where previously found lie outside the clock regime. They are eigenstate of $\hat{H}$ whith vanishing eigenvalue. Classically, the vacuum solutions of the theory are only compatible with $\Pi=0$, namely $\dot{T}=0$, which clearly indicates that we are outside th eregime in which we can take the scalar field as a good clock.

We do not consider this necessary restriction of the formalism as a serious limitation. Our long term aim is to develop a usable theory that can be employed, at least in principle, to describe Planck scale measurements and the Planck scale evolution of quantum geometry. We would be very content of having a sensible general covariant field theory that correctly describe this physics in the regime in which whatever we are using as a clock keeps behaving as a clock.

4. Scalar product. One of the weak points of the Loop Representation is given by the fact that a complete and consistent definition of the scalar product is not yet available. The conventional wisdom is that once physical observables on the physical state space have been constructed, the scalar product is determined by the requirement these physical observables be self-adjoint. The present work is a step in this direction. The (real eigenvalues) states of the hamiltonian $\hat{H}$ must form, if the formalism is consistent, an orthogonal basis. Thus, working out explicitely the eigenstates of $\hat{H}$ in knot space should at the same time lead to a partial definition of the scalar product. For a discussion of the definition of the scalar product for fermion in functional representations, see [35].

5. Taking limits on knot space. Finally, let us discuss a subtle point in the definition of $\hat{H}$, which we percieve as the most delicate and potentially problematic point in the construction above. We refer to the different way in which the $\delta \rightarrow 0$ and the $\tau \rightarrow 0$ limit have been dealt with, when dealing with knot states.

To focus the point, let us consider a model example. Consider the space $C[R]$ of the continuous functions $f(x)$ on the real line. Consider the closure $D$ of the space $C[R]$, say in the pointwise topology, such that $D$ contains also piecewise continuous functions as the step function $\theta$ defined by

$$
\theta(x)=1 \text { if } x>0 ; \theta(x)=0 \text { otherwise. }
$$

Now define the linear functional $k$ on $C[R]$ as follows: $(k, f):=\lim _{x \rightarrow 0} f(x)$, and assume you want to extend $k$ from $C[R]$ to $D$. There are two possible strategies: one is to keep the definition

$$
(k, f)=\lim _{x \rightarrow 0} f(x) .
$$


The other is to note that an equivalent definition of $k$ on $C[R]$ is $(k, f)=$ $f(0)$, and thus to define

$$
(k, f)=f(0) .
$$

According to the first definition

$$
(k, \theta)=1,
$$

according to the second

$$
(k, \theta)=0 .
$$

We are in a similar situation when we need to study the action of the hamiltonian $\hat{H}$ on knot states. In quantum mechanics, operators are often defined on dense subspaces of the state space. For instance we begin by defining the momentum operator in Schrödinger mechanics not on the full $L_{2}$ state space but on the dense subspace of the differentiable functions; then we can extend it. The Hamiltonian $\hat{H}$ that we define in this paper contains a certain number of limiting procedures. We may first rigourously define it on a suitable restricition of the space of the loop functionals. For instance we may assume that the loop functionals are continuous in all the deformations that we consider. $\hat{H}$ is well defined on this space. Then, however, we want to consider the action of $\hat{H}$ on the knot states. These are not continuous in the deformations that we consider and thus we need to define a suitable extension of the operator. At this point we have a choice that essentially reflects the choice we described in the simple example above. As far as we understand, this choice, if not dictated by internal consistency, is again part of the quantization ambiguities as the ordering of the dynamical operators.

The important point we want to make here is not that a choice of the extension has been made in computing the action of $\hat{H}$ on the knot states, but that two different choices have been made for the two limits $\delta \rightarrow 0$ and $\tau \rightarrow 0$. In fact, as far as the $\delta \rightarrow 0$ is concerned, we have assumed that we should first take the limit, and then consider the extension of the action of the operator to diffeomorphism invariant states; while as far as the $\tau \rightarrow 0$ limit is concerned, we have assumed that we should first extend the the operator to diffeomorphism invariant states, and then take the limit.

This choice is not completely arbitrary: $\delta$ must go to zero faster that $\tau$, and, if we take away the fake dimensions added by the integration, we see that the first significative term, which is the one that we are considering, is of order zero in $\delta$ and of first order in $\tau$. Ths means that already at the classical level what we are doing is precisely considering a function $f(\delta, \tau)$ and picking up terms of the form $\left.\frac{\partial}{\partial \tau} f(\delta, \tau)\right|_{\delta=\tau=0}$. Thus it is not completely unreasonable that this difference gets translated in the different ways in which the two limits are taken on loop space: roughly, we are 
"really" looking at the $\delta=0$ point, and we are "really" looking at the limit in the first order expansion in $\tau$. However, these are hand-waving justifications of our choice. Untill a well-defined calculus on Loop Space is constructed [36], we do not see a way to transform these tentative explorations into solid mathematics. Our only real justification at this point, if any, is the hope that the (finite) structure we are constructing be internally consistent and, perhaps, related to Nature.

\subsection{What next}

The next step in the construction of the theory should be to compute explicitely matrix elements of $\hat{M}_{i}$ and $\hat{F}_{e}$ (see eq.(90)), and to understand whether there is a direct algoritm for extracting the square root. If this can be done, the theory is essentially at the stage where the evolution of physical states can be described.

As noted above, the scalar product is partially fixed by the construction itself. The energy and the particle number are conserved observables. There are other observables in the theory that one may consider, and evolve, as the area observable discussed in references [2, 1. A crucial test for the consistency of the scheme developed here is, as was noted in [5], whether the second order term of the time dependent perturbation expansion develops divergences.

We are deeply indebited with Lee Smolin for ideas, criticism, and encouragement. This work was partially supported by the NSF Grant PHY-9311465.

\section{References}

[1] C. Rovelli and L. Smolin, Phys. Rev. Lett. 61, 1155 (1988); Nucl. Phys. B133 (1990) 80.

[2] A. Ashtekar, C. Rovelli, L. Smolin, Phys Rev Lett 69 (1992) 237

[3] J Iwasaki, C. Rovelli, : "Gravitons as Embroidery on the Weave", International Journal of Modern Physics D1, 533 (1993); "Gravitons from loops: non-perturbative loop-space quantum gravity contains the graviton-physics approximation", submitted to Classical and Quantum Gravity.

[4] C. Rovelli, A generally covariant quantum field theory and a prediction on quantum measurements of geometry, Nuclear Physics B405, 797 (1993).

[5] Rovelli C and Smolin L 1993 "The physical hamiltonian in non- perturbative quantum gravity" Pittburgh University Preprint (1993) gr-qg?.

[6] C. Rovelli, Classical and Quantum Gravity, 8 (1991) 1613. 
[7] A. Ashtekar, Non-perturbative canonical gravity. Lecture notes prepared in collaboration with R. S. Tate. (World Scientific, Singapore,1991).

[8] L. Smolin, Recent developments in nonperturbative quantum gravity in the Proceedings of the 1991 GIFT International Seminar on Theoretical Physics: Quantum Gravity and Cosmology, (World Scientific, Singapore, in press).

[9] C. Rovelli: "What is observable in classical and quantum gravity?", Classical and Quantum Gravity 8, 297 (1991).

[10] C. Rovelli: "Quantum reference systems", Classical and Quantum Gravity 8, 317 (1991).

[11] A. Einstein, Über die spezielle und die allgemeine Relativitätstheorie , (Vieweg, Braunshweig, 1920); P. G. Bergmann, Helv. Phys. Acta Suppl. 4, 79 (1956); J. Stachel, Einstein search for general covariance 1912- 1915 in Einstein and the History of General Relativity eds. D.Howard and J. Stachel, Einstein studies, Volume 1, Birkhouser, Boston, 1989. B. S. DeWitt, in Gravitation, An Introduction to Current Research ed. L. Witten (Wiley, New York,1962).

[12] R. Gambini and A. Trias, Phys. Rev. D23 (1981) 553, Lett. al Nuovo Cimento 38 (1983) 497; Phys. Rev. Lett. 53 (1984) 2359; Nucl. Phys. B238, 436 (1986); B278 (1986) 436; R. Gambini, L. Leal and A. Trias, Phys. Rev. D39 (1989) 3127; R. Gambini, Phys. Lett. B 255 (1991) 180. R. Gambini and L. Leal, Loop space coordinates, linear representations of the diffeomorphism group and knot invariants preprint, University of Montevideo, 1991. $S U$ (2) QCD in the path representation, University of Montevideo preprint 1993.

[13] Dirac Sciama

[14] Nelson

[15] A. Ashtekar. New variables for classical and quantum gravity. Physical Review Letters 57, 2244-2247 (1986). New Hamiltonian formulation of general relativity. Physical Review D36, 1587-1602 (1987).

[16] A. Ashtekar, J. D. Romano, and R. S. Tate. New variables for gravity: Inclusion of matter. Phys.Rev.D D40, 2572-2587 (1989).

[17] K. Wilson, Phys. Rev. D10, 247 (1974); J. Kogut, L. Suskind, Phys. Rev. D11, 395 (1975); L. Susskind Coarse grained quantum chromodynamics in it Les Houches XXIX Weak and Electromagnetic Interactions at High Energy ed. R. Balian and C.H. Llewellyn-Smith (Amsterdam:North Holland).

[18] H. Morales-Tecotl. Ph.D. thesis 1993. Unpublished. 
[19] C. Rovelli, in Conceptual Problems of Quantum Gravity ed. A. Ashtekar and J. Stachel, (Birkhauser,Boston,1991); Phys. Rev. D 42 (1991) 2638; 43 (1991) 442.

[20] C. Rovelli: "Time in quantum gravity: an hypothesis", Physical Review D43, 442 (1991).

[21] C. Rovelli : "Quantum mechanics without time: a model" Physical Review D42, 2638 (1991); "Quantum evolving constants", Physical Review D44, 1339 (1991).

[22] K. Kuchar and C. Torre, Phys. Rev. D43 (1991) 419; D44 (1991) 3116. L. Smolin, in the Brill Feschrift Proceedings, ed. B-l Hu, T. Jacobson, (CUP, Cambridge,1993). K. Kuchar, in the Brill Feschrift Proceedings op . cit..

[23] A. V. Ashtekar, Phys. Rev. Lett. 57, 2244 (1986) ; Phys. Rev. D 36 (1987) 1587.

[24] esposito morales kim

[25] esposito morales

[26] T. Jacobson and L. Smolin. The left-handed spin connection as a variable for canonical gravity. Physics Letters B196, 39-42 (1987). J. Samuel. A Lagrangian basis for Ashtekar's reformulation of canonical gravity. PramānaJ Phys. 28, L429-L432 (1987). T. Jacobson and L. Smolin. Covariant action for Ashtekar's form of canonical gravity. Classical and Quantum Gravity 5, 583-594 (1988)

[27] T. Jacobson. Fermions in canonical gravity. Class. and Quantum Grav. 5(10):L143-L148, October 1988.

[28] A. Ashtekar, L. Bombelli and O. Reula, In: Analysis, Geometry and Mechanics: 200 Years after Lagrange, eds. M. Francaviglia and D. Holm (North Holland, Amsterdam, in press).

[29] C. Rovelli, An introduction to canonical Quantum Gravity. Lecture notes, 1992, Unpublished.

[30] Kim

[31] S. Mandelstam, Ann. Phys. 19 (1962) 1. A. M. Polyakov, Phys. Lett. 82B, 247 (1979); Nucl. Phys. B164, 171 (1979). Yu.M. Makeenko, A.A. Migdal Phys. Lett. 88B, 135 (1979). Y. Nambu, Phys. Lett. 80B, 372 (1979); F. Gliozzi, T. Regge, M.A. Virasoro, Phys. Lett. 81B, 178 (1979); M. Virasoro, Phys. Lett. 82B, 436 (1979); A. Jevicki, B. Sakita, Phys. Rev. D22, 467 (1980); B. Sakita, "Collective field theory" in "Field theory in elementary particles" ed. B. Kursunoglu and A. Perlmutter, Plenum Publishing Coprporation, 1983. 
[32] C. Rovelli, L. Smolin: "Loop representation for Yang Mills theories: Lattice". Roma preprint (1989).

[33] B Brügmann, Phys Rev D 43, 566 (1991).

[34] C. J. Isham, in Relativity, Groups and Topology II, ed B.S. DeWitt and R. Stora (Amsterdam, Elsevier).

[35] For a review of functional representations of quantum field theory (including fermions), see J H Yee in "Recent developments in field theory", ed J E Kim, Mineum Publishing Co 1992. Functional representations for fermions have been studied by R. Floreanini, R. Jackiw, Phys. Rev. D37, 2206 (1988). A. Duncan, H. Meyer-Ortmanns, R. Roskies, Phys. Rev. D36, 3788 (1987).

[36] A. Ashtekar, C.J. Isham, Class. and Quant. Grav. 9, 1069 (1992). A. Ashtekar, J Lewandowski, in "Knots and Quantum Gravity", ed J Baez, Oxford University Press (1993).

[37] MP Blencowe, Nucl. Phys. B341, 213 (1990). V. Husain, Hamiltonian constraint of quantum general relativity, Nucl. Phys. B313, 711-724 (1988). B. Brügmann and J. Pullin, Nucl. Phys. B363, 221 (1991).

[38] B Brügmann, The Hamiltonian Constraint in the Loop Representation. Ph.D. Thesis 1992. Unpublished. 